\title{
Synthesis of novel N-, S-substituted-polyhalo-1, 3-butadienes and crystal structure of dibutadienyl homopiperazine
}

\author{
NAHIDE GULSAH DENIZ and CEMIL IBIS* \\ Engineering Faculty, Department of Chemistry, Division of Organic Chemistry, Istanbul University, Avcilar, \\ Istanbul, Turkey \\ e-mail: ibiscml@istanbul.edu.tr
}

MS received 4 November 2012; revised 18 February 2013; accepted 5 April 2013

\begin{abstract}
Polyhalogenated-2-nitro-1, 3-butadienes are important synthetic precursors for a variety of polyfunctionalized bioactive heterocycles. Herein, we report the reactions of 1, 1, 3, 4, 4-pentachloro-2-nitro-1, 3-butadiene 1 and 4-bromo-1, 1, 3, 4-tetrachloro-2-nitro-1, 3-butadiene 2 with amino and thiol containing nucleophiles to obtain highly functionalized (E)-polyhalodiene-2-nitro-1, 3-butadiene derivatives. Most of these reactions were found to be highly selective resulting in good to high yields of the products. All new compounds have been characterized by nuclear magnetic resonance spectroscopy (NMR), mass spectrometry (MS) and Fourier transform infrared spectroscopy (FT-IR) spectroscopic data. Single crystal X-ray structure analysis of compound $\mathbf{8 c}$ is reported.
\end{abstract}

Keywords. Piperazine; homopiperazine; thiomorpholine; 1, 3-butadiene; $E$-isomers; X-ray structure.

\section{Introduction}

Nitro-substituted polyhalogeno-1, 3-butadienes have proven to be valuable synthetic precursors for a variety of polyfunctionalized bioactive heterocycles due to their stepped reactivity in $\mathrm{S}_{\mathrm{N}}$ reactions. ${ }^{1}$ Often, the building block of choice is 2-nitroperchloro-1, 3butadiene $(\mathbf{1}, \mathbf{2})$, which is easily accessible by the introduction of an activating and directing nitro group into $2 \mathrm{H}$-pentachloro-1, 3-butadiene. Synthetic use of $(\mathbf{1}, \mathbf{2})$ opens access to a quite diverse chemistry, the documentation of which was started by our group recently. ${ }^{1,2}$ The preferred primary reaction centre of $(\mathbf{1}, \mathbf{2})$ is the activated terminal carbon atom $\mathrm{C}-1$ of the nitrodichlorovinyl moiety. This carbon atom allows for an attack by different nucleophiles in $\mathrm{S}_{\mathrm{N}}$ Vin processes. Under harsher conditions, the internal carbon atom, C-3, is additionally open to the attack of nucleophiles.

In literature, many studies are reported on the biodegradation of morpholine with strains of Mycobacterium or Arthrobacter and Gram negative bacteria. ${ }^{3-6}$ Thiomorpholine analogues have found applications in medicine and agriculture. ${ }^{7} \mathrm{~N}$-arylpiperazines, $\mathrm{N}$ arylindoles, $N$-arylpyrroles and substituted anilines are important compounds, particularly, in pharmaceutical research and also as intermediates of synthesis. The

*For correspondence
$N$-arylpiperazine subunit is embedded in several pharmacologically interesting targets such as compounds related to the serotonine ligands, calcium blockers, antipsychotic drugs, antihypertensive and acetylcholinesterase inhibitory activity. ${ }^{8-12}$

We have reported that some N-, S-, O-substituted polyhalo-2-nitro-1, 3-butadiene compounds were synthesized from reactions of 1, 1, 3, 4, 4-pentachloro2-nitro-1, 3-butadiene $\mathbf{1}$ and 1, 1, 3, 4-tetrachloro4-bromo-2-nitro-1, 3-butadiene $\mathbf{2}$ with $\mathrm{N}-$, $\mathrm{S}$ nucleophiles. ${ }^{13-17}$ In this study, we present the results of various reactions of 1, 1, 3, 4, 4-pentachloro-, 1, 1, 3, 4-tetrachloro-4-bromo-, and also 1-mono(alkylthio)polyhalo-2-nitro-1, 3-butadiene with S-, S, S-, and N, $\mathrm{S}$ - nucleophiles as well as some additional conversions of the resulting compounds and characterization of their structures by using micro-analysis, Fourier transform infrared spectroscopy (FT-IR), ${ }^{1} \mathrm{H}$-nuclear magnetic resonance spectroscopy (NMR), ${ }^{13} \mathrm{C}-\mathrm{NMR}$ and mass spectrometry (MS). In addition, single crystal structure of compound $\mathbf{8} \mathbf{c}^{18}$ was solved by using X-ray diffraction method.

\section{Experimental}

\subsection{General apparatus}

Melting points were measured on a Buchi B-540 melting point apparatus. Elemental analyses were 
performed on a Thermo Finnigan Flash EA 1112 Elemental analyser. Infrared (IR) spectra were recorded in $\mathrm{KBr}$ pellets in Nujol mulls on a Perkin Elmer Precisely Spectrum One FT-IR spectrometry. ${ }^{1} \mathrm{H}$ and ${ }^{13} \mathrm{C}$ NMR spectra were recorded on Varian UNITYINOVA operating at $500 \mathrm{MHz}$. Chemical shifts $\delta(\mathrm{ppm})$ are reported relative to tetramethylsilane (TMS) with the solvent resonance employed as the internal standard. ${ }^{1} \mathrm{H}$ and ${ }^{13} \mathrm{C}$ NMR spectra in $\mathrm{CDCl}_{3}$ refer to the solvent signal centre at $\delta=7.26 \mathrm{ppm}$ and $\delta=77.0 \mathrm{ppm}$, respectively. Mass spectra were obtained on a Thermo Finnigan LCQ Advantage MAX LC/MS/MS spectrometer according to electron spray ionization (ESI) probe. Products were isolated by column chromatography on silica gel (Fluka Silica gel 60, particle size 63-200 $\mu \mathrm{m}$ ). Analytical thin-layer chromatography (TLC) was purchased from Merck KGaA (silica gel 60 F254) based on Merck DC-plates (aluminum-based). Visualization of the chromatogram was performed by UV light $(254 \mathrm{~nm})$. All chemicals were reagent grade and used without further purification. Moisture was excluded from the glass apparatus using $\mathrm{CaCl}_{2}$ drying tubes. Solvents, unless otherwise specified, were of reagent grade and distilled once prior to use.

\subsection{Crystal structure determination and refinement}

Yellow crystals of compound $\mathbf{8} \mathbf{c}^{18}$ suitable for X-ray diffraction analysis were obtained by slow evaporation of ethanol solution at room temperature. A yellow single crystal of compound $8 c, \mathrm{C}_{21} \mathrm{H}_{28} \mathrm{Cl}_{6} \mathrm{~N}_{4} \mathrm{O}_{4} \mathrm{~S}_{2}$, having approximate dimensions of $0.60 \times 0.10 \times 0.10 \mathrm{~mm}$ was mounted on a glass fibre. All measurements were made on a Rigaku R-Axis Rapid-S imaging plate area detector with graphite monochromated Mo-K $\alpha$ radiation $(\lambda=0.71073 \AA)$. The data were collected at room temperature to a maximum $2 \theta$ value of $50.2^{\circ}$. Experimental conditions are summarized in table 1 . The structure was solved by SIR $92{ }^{19}$ and refined with CRYSTALS. ${ }^{20}$ The non-hydrogen atoms were refined anisotropically. $\mathrm{H}$ atoms were located in geometrically idealized positions $\mathrm{C}-\mathrm{H}=0.95(6) \AA$ and treated as riding and $U_{\text {iso }}(\mathrm{H})=1.2 U_{\text {eq }}(\mathrm{C})$. The selected bond distances, bond and torsion angles for compound $\mathbf{8 c}$ are listed in tables 2 and 3, respectively. Drawings were performed with the program ORTEP-III ${ }^{21}$ with $50 \%$ probability displacement ellipsoide for compound 8c in figure 1. Crystallographic data (excluding structure factors) for the structure reported in this study have been deposited with the Cambridge Crystallographic Data Centre as supplementary publication no. CCDC-907293 for $8 \mathbf{c} .^{22}$
Table 1. Crystal data and refinement parameters for compound $\mathbf{8 c}$.

\begin{tabular}{ll} 
CCDC deposit number & $\mathrm{CCDC} 907293$ \\
Empirical formula & $\mathrm{C}_{21} \mathrm{H}_{28} \mathrm{Cl}_{6} \mathrm{~N}_{4} \mathrm{O}_{4} \mathrm{~S}_{2}$ \\
Crystal colour, habit & Yellow, chunk \\
Formula weight & 677.31 \\
Temperature & $293(2) \mathrm{K}$ \\
Wavelength & $0.71073 \AA$ \\
Crystal system & Monoclinic \\
Space group & $\mathrm{P} 21 / n$ \\
Cell dimensions & $a=7.9290(6) \AA$ \\
& $b=12.4335(8) \AA$ \\
& $c=31.167(2) \AA$ \\
& $\beta=95.504(4)^{\circ}$ \\
Volume & $3058.5(4) \AA^{3}$ \\
$Z$ & 4 \\
Density (calculated) & $1.471 \mathrm{mg} / \mathrm{m}^{3}$ \\
Absorption coefficient & $0.731 \mathrm{~mm}{ }^{-1}$ \\
$F_{000}$ & 1392.00 \\
Index ranges & $-9 \leq h \leq 8$ \\
& $-14 \leq k \leq 14$ \\
Reflections collected & $-37 \leq l \leq 36$ \\
Independent reflections & 24008 \\
Data/restraints/parameters & $5444\left[R_{\text {int }}=0.083\right]$ \\
Goodness of fit indicator & $4570 / 0 / 334$ \\
Final $R$ indices $[I>2 \sigma(I)]$ & 1.266 \\
Largest difference peak and hole & 0.54 and $-0.47 e . \AA^{-3}$ \\
\hline &
\end{tabular}

Table 2. Selected bond distances $(\AA)$ for compound $\mathbf{8 c}$.

\begin{tabular}{lcrr}
\hline Atom & Distance $(\AA)$ & \multicolumn{1}{c}{ Atom } & Distance $(\AA)$ \\
\hline C1-C2 & $1.29(1)$ & N2-C13 & $1.47(1)$ \\
C2-C3 & $1.44(1)$ & C12-C13 & $1.51(1)$ \\
C3-C4 & $1.43(2)$ & N3-C12 & $1.49(1)$ \\
N2-C9 & $1.47(1)$ & C14-C15 & $1.43(2)$ \\
C9-C10 & $1.52(1)$ & C15-C16 & $1.42(2)$ \\
C10-C11 & $1.51(1)$ & C16-C17 & $1.30(2)$ \\
\hline
\end{tabular}

\subsection{Synthesis of $S$-, $S, S$-, $S, S, S$ - and $N, S$-substitute nitrodienes}

Method 1: Equimolar amounts of polyhalo-2nitrodiene (1 and $\mathbf{2}$ ) and thiols were mixed directly or in dichloromethane and stirred between $12-48 \mathrm{~h}$ at room temperature. The yields of reaction were extracted with chloroform $(20 \mathrm{~mL})$ and water $(20 \mathrm{~mL})$ and dried with sodium sulphate $\left(\mathrm{Na}_{2} \mathrm{SO}_{4}\right)$. The residues were purified by column chromatography on silica gel.

Method 2: Polyhalo-2-nitrodiene (1 and 2) and three molar equivalent thiols were dissolved in ethanol ( $25 \mathrm{~mL}$ ) and added to the solution of sodium hydroxide in ethanol and stirred between 12 and $48 \mathrm{~h}$ at room 
Table 3. Selected bond and torsion angles $\left(^{\circ}\right)$ for compound 8c.

\begin{tabular}{lrlr}
\hline Atom & Bond angle $\left({ }^{\circ}\right)$ & \multicolumn{1}{c}{ Atom } & Torsion angle $\left(^{\circ}\right)$ \\
\hline C1-C2-C3 & $125.0(9)$ & C1-C2-C3-C4 & $-56.0(1)$ \\
C2-C3-C4 & $122.3(9)$ & C14-C15-C16-C17 & $64.0(1)$ \\
C14-C15-C16 & $122.0(1)$ & C1-C2-C3-N1 & $127.0(1)$ \\
C15-C16-C17 & $123.0(1)$ & C17-C16-C15-N4 & $-114.0(1)$ \\
C9-N2-C13 & $113.7(8)$ & N2-C9-C10-C11 & $91.0(1)$ \\
C11-N3-C12 & $115.2(8)$ & N2-C13-C12-N3 & $-68.0(1)$ \\
C9-C10-C11 & $113.6(8)$ & C5-C6-C7-C8 & $174.0(1)$ \\
O1-N1-O2 & $122.9(9)$ & C18-C19-C20-C21 & $-168.0(2)$ \\
\hline
\end{tabular}

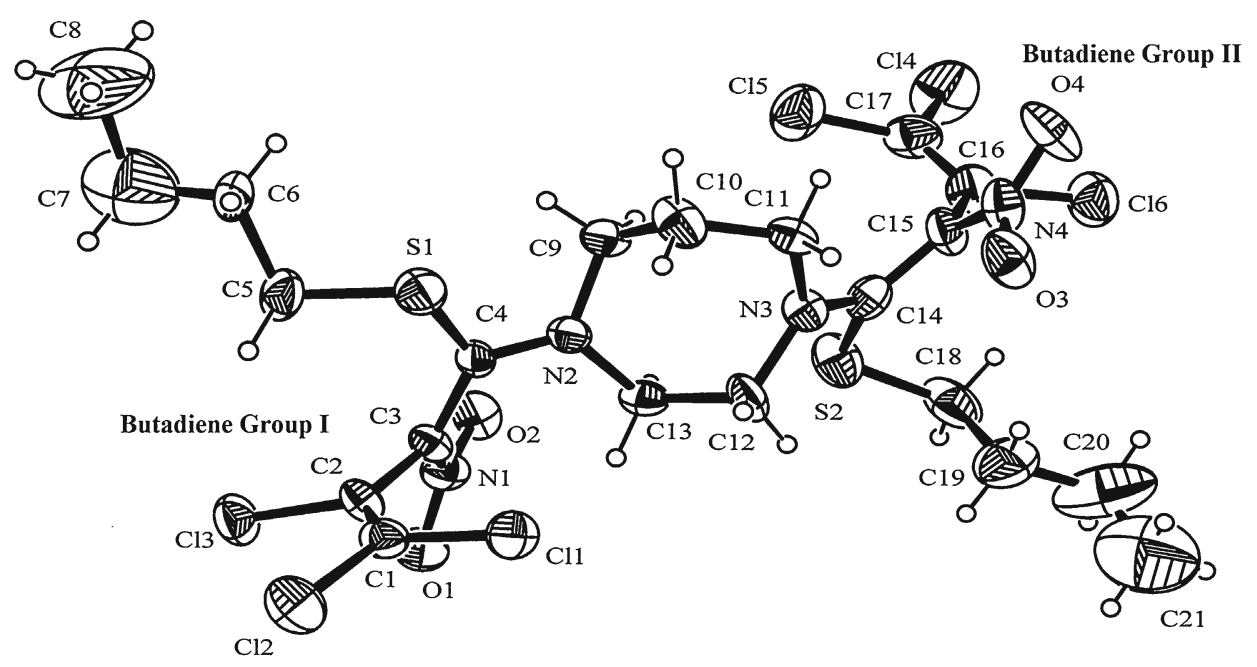

Figure 1. Molecular structure of the compound 8c. Displacement ellipsoids are drawn at 50\% probability level.

temperature. The yields of reaction were extracted with chloroform $(20 \mathrm{~mL})$ and water $(20 \mathrm{~mL})$ and dried with sodium sulphate $\left(\mathrm{Na}_{2} \mathrm{SO}_{4}\right)$. After the solvent was evaporated, the residue was purified by column chromatography on silica gel.

Method 3: S-monothiosubstituted-2-nitro-1, 3-butadiene and thiomorpholine and some piperazine derivatives were dissolved in dichloromethane $(25 \mathrm{~mL})$ and stirred between 12 and $48 \mathrm{~h}$ at room temperature. The yields of reaction were extracted with chloroform $(20 \mathrm{~mL})$ and water $(20 \mathrm{~mL})$ and dried with sodium sulphate $\left(\mathrm{Na}_{2} \mathrm{SO}_{4}\right)$. The residues were purified by column chromatography on silica gel.

2.3a 1, 3, 4, 4-Tetrachloro-2-nitro-(1E)-(pentylsulphanyl)1, 3-butadiene $(\mathbf{4 d})$ : Compound $\mathbf{4 d}$ was synthesized from reaction of $\mathbf{1}(2 \mathrm{~g}, 7.37 \mathrm{mmol})$ with pentanethiol 3d $(0.76 \mathrm{~g}, 7.37 \mathrm{mmol})$ according to method 1. Yield: $1.88 \mathrm{~g}$ (75\%); Yellow oil. $R_{\mathrm{f}}$ $\left(\mathrm{CCl}_{4}\right): 0.41 ; \mathrm{IR}\left(\mathrm{KBr}, \mathrm{cm}^{-1}\right): v=2874,2935,2965$ $(\mathrm{C}-\mathrm{H}), 1462,1605(\mathrm{C}=\mathrm{C}), 1292,1535\left(\mathrm{C}-\mathrm{NO}_{2}\right)$; ${ }^{1} \mathrm{H} \mathrm{NMR}\left(499.74 \mathrm{MHz}, \mathrm{CDCl}_{3}\right): \delta=0.8(\mathrm{t}, J=6.83 \mathrm{~Hz}$, $\left.3 \mathrm{H}, \mathrm{CH}_{3}\right), 1.0-1.5\left(\mathrm{~m}, 4 \mathrm{H}, 2 \mathrm{CH}_{2}\right), 1.7(\mathrm{~m}, 2 \mathrm{H}, \mathrm{S}-$ $\left.\mathrm{CH}_{2}-\mathrm{CH}_{2}\right), 3.1 \mathrm{ppm}\left(\mathrm{t}, J=7.32 \mathrm{~Hz}, 2 \mathrm{H}, \mathrm{S}-\mathrm{CH}_{2}\right)$. ${ }^{13} \mathrm{C}$ NMR $\left(125.66 \mathrm{MHz}, \mathrm{CDCl}_{3}\right): \delta=14.08\left(\mathrm{CH}_{3}\right)$, 22.39, $28.32\left(\mathrm{CH}_{2}\right), 29.93\left(\mathrm{~S}-\mathrm{CH}_{2}-\mathrm{CH}_{2}\right), 31.08(\mathrm{~S}-$ $\left.\mathrm{CH}_{2}\right), 121.86,128.80,138.77,158.37 \mathrm{ppm}\left(\mathrm{C}_{\text {butad }}\right)$. Anal. $\mathrm{C}_{9} \mathrm{H}_{11} \mathrm{~N}_{1} \mathrm{O}_{2} \mathrm{~S}_{1} \mathrm{Cl}_{4}(M=339.07 \mathrm{~g} / \mathrm{mol})$. Micro analysis: Calcd. C, 31.88; H, 3.27; N, 4.13; S, 9.45. Found C, 31.89; H, 3.25; N, 4.14; S, 9.48\%.

2.3b 1, 3, 4, 4-Tetrachloro-2-nitro-(1E)-(2-ethyl-hexylsulphanyl)-1, 3-butadiene (4h): Compound $4 \mathrm{~h}$ was synthesized from reaction of $\mathbf{1}(2 \mathrm{~g}, 7.37 \mathrm{mmol})$ with 2-ethyl-hexanethiol $3 \mathbf{h}(1.07 \mathrm{~g}, 7.37 \mathrm{mmol})$ according to method 1 . Yield: $1.85 \mathrm{~g}(66 \%)$; Yellow oil. $R_{\mathrm{f}}$ $\left(\mathrm{CCl}_{4}\right): 0.41 ; \mathrm{IR}\left(\mathrm{KBr}, \mathrm{cm}^{-1}\right): v=2960,2929,2872$, $2859(\mathrm{C}-\mathrm{H}), 1460,1603(\mathrm{C}=\mathrm{C}), 1292,1537(\mathrm{C}-$ $\left.\mathrm{NO}_{2}\right) ;{ }^{1} \mathrm{H}$ NMR $\left(499.74 \mathrm{MHz}, \mathrm{CDCl}_{3}\right): \delta=0.8-0.9(\mathrm{t}$, $\left.J=6.83 \mathrm{~Hz}, 6 \mathrm{H}, 2 \mathrm{CH}_{3}\right), 1.2-1.4\left(\mathrm{~m}, 8 \mathrm{H}, 4 \mathrm{CH}_{2}\right)$, $1.7\left(\mathrm{~m}, 1 \mathrm{H}, \mathrm{S}-\mathrm{CH}_{2}-\mathrm{CH}\right), 3.0 \mathrm{ppm}\left(\mathrm{d}, 2 \mathrm{H}, \mathrm{S}-\mathrm{CH}_{2}\right)$. ${ }^{13} \mathrm{C}$ NMR $\left(125.66 \mathrm{MHz}, \mathrm{CDCl}_{3}\right): \delta=13.00,13.02$ $\left(\mathrm{CH}_{3}\right), 21.82,24.65,27.66,27.75\left(\mathrm{CH}_{2}\right), 31.24$ $\left(\mathrm{S}-\mathrm{CH}_{2}-\mathrm{CH}\right), 37.66\left(\mathrm{~S}-\mathrm{CH}_{2}\right), 120.65,127.55,137.72$, $157.22 \mathrm{ppm}\left(\mathrm{C}_{\text {butad }}\right)$. Micro analysis: $\mathrm{C}_{12} \mathrm{H}_{17} \mathrm{~N}_{1} \mathrm{O}_{2} \mathrm{~S}_{1} \mathrm{Cl}_{4}$ 
$(M=381.151 \mathrm{~g} / \mathrm{mol})$. Calcd. C, 37.80; H, 4.49; N, 3.67; S, 8.41. Found C, 37.89; H, 4.45; N, 3.64; S, 8.48\%.

2.3c 3, 4, 4-Trichloro-2-nitro-(1E)-[pentylsulphanyl4-(thiomorpholin-1-yl)]-1, 3-butadiene (5d): Compound $5 \mathbf{d}$ was synthesized from reaction of $\mathbf{4 d}(0.5 \mathrm{~g}$, $1.47 \mathrm{mmol})$ with thiomorpholine $(0.15 \mathrm{~g}, 1.47 \mathrm{mmol})$ according to method 3. Yield: $0.48 \mathrm{~g}(81 \%)$; Yellow oil. $R_{\mathrm{f}}\left(\mathrm{CCl}_{4}\right): 0.48 ; \mathrm{IR}\left(\mathrm{KBr}, \mathrm{cm}^{-1}\right): v=2857,2928$, $2858(\mathrm{C}-\mathrm{H}), 1522,1652(\mathrm{C}=\mathrm{C}), 1270,1522\left(\mathrm{C}-\mathrm{NO}_{2}\right)$; ${ }^{1} \mathrm{H}$ NMR $\left(499.74 \mathrm{MHz}, \mathrm{CDCl}_{3}\right): \delta 0.85(\mathrm{t}, J=$ $\left.7.32 \mathrm{~Hz}, 3 \mathrm{H}, \mathrm{CH}_{3}\right), 1.2-1.4\left(\mathrm{~m}, 4 \mathrm{H}, \mathrm{CH}_{2}\right), 1.6(\mathrm{~m}$, $\left.2 \mathrm{H}, \mathrm{S}-\mathrm{CH}_{2}-\mathrm{CH}_{2}\right), 2.90$ (t, $J=5.37 \mathrm{~Hz}, 2 \mathrm{H}, \mathrm{S}-\mathrm{CH}_{2}$ ), $2.65\left(\mathrm{t}, J=4.88 \mathrm{~Hz}, 4 \mathrm{H}, \mathrm{H}_{\text {thiomorp}}\right), 3.40 \mathrm{ppm}(\mathrm{s}$, br, $\left.4 \mathrm{H}, \mathrm{H}_{\text {thiomorp }}\right) ;{ }^{13} \mathrm{C} \mathrm{NMR}\left(125.66 \mathrm{MHz}, \mathrm{CDCl}_{3}\right)$ : $\delta 12.80\left(\mathrm{CH}_{3}\right), 21.11,26.87\left(\mathrm{CH}_{2}\right), 29.82\left(\mathrm{~S}-\mathrm{CH}_{2}-\right.$ $\left.\mathrm{CH}_{2}\right), 34.42\left(\mathrm{~S}-\mathrm{CH}_{2}\right), 43.46,54.98\left(\mathrm{C}_{\text {thiomorp }}\right), 118.17$, 123.81, 132.87, $169.60 \mathrm{ppm}\left(\mathrm{C}_{\text {butad }}\right)$; Micro analysis: $\mathrm{C}_{13} \mathrm{H}_{19} \mathrm{~N}_{2} \mathrm{O}_{2} \mathrm{~S}_{2} \mathrm{Cl}_{3}$ (M, 405.79). Calcd. C, 38.47; H, $4.71 ; \mathrm{N}, 6.90 ; \mathrm{S}, 15.80$ Found $\mathrm{C}, 38.24 ; \mathrm{H}, 4.64 ; \mathrm{N}$, $6.80 ; \mathrm{S}, 15.85 \%$.

2.3d 3, 4, 4-Trichloro-2-nitro-(1E)-[ethylsulphanyl-1(1-piperonylpiperazin-1-yl)]-1, 3-butadiene (7a): Compound 7a was synthesized from reaction of $4 \mathbf{a}(0.5 \mathrm{~g}$, $1.68 \mathrm{mmol})$ with 1-(piperonyl)piperazine $\mathbf{6 a}(0.37 \mathrm{~g}$, $1.68 \mathrm{mmol})$ according to method 3 . Yield: $0.52 \mathrm{~g}$ (64\%); m.p.: $228-229^{\circ} \mathrm{C} . R_{\mathrm{f}}: 0.65\left(\mathrm{CH}_{2} \mathrm{Cl}_{2}\right)$. IR ( $\mathrm{KBr}$, $\left.\mathrm{cm}^{-1}\right): v=3053(\mathrm{Ar}-\mathrm{H}), 2925,2852,2809,2771$ $(\mathrm{C}-\mathrm{H}), 1523,1596(\mathrm{C}=\mathrm{C}), 1289,1450\left(\mathrm{C}-\mathrm{NO}_{2}\right)$. ${ }^{1} \mathrm{H}-\mathrm{NMR}\left(499.74 \mathrm{MHz}, \mathrm{CDCl}_{3}\right): \delta=1.18(\mathrm{t}, J=$ $\left.7.81 \mathrm{~Hz}, 3 \mathrm{H}, \mathrm{CH}_{3}\right), 2.5\left(\mathrm{sbr}, 4 \mathrm{H}, \mathrm{H}_{\text {piper }}\right), 2.9(\mathrm{t}, J=$ $\left.7.32 \mathrm{~Hz}, 2 \mathrm{H}, \mathrm{S}-\mathrm{CH}_{2}\right), 3.4\left(\mathrm{~s}, 2 \mathrm{H}, \mathrm{N}-\mathrm{CH}_{2}\right), 3.6$ (sbr, $\left.4 \mathrm{H}, \mathrm{H}_{\text {piper }}\right), 5.88$ (s, 2H, O- $\left.\mathrm{CH}_{2}-\mathrm{O}\right), 6.6-6.9 \mathrm{ppm}(\mathrm{m}$, $\left.3 \mathrm{H}, \mathrm{H}_{\text {arom }}\right) .{ }^{13} \mathrm{C}-\mathrm{NMR}\left(125.66 \mathrm{MHz}, \mathrm{CDCl}_{3}\right): \delta=$ $13.90\left(\mathrm{CH}_{3}\right), 28.74\left(\mathrm{~S}-\mathrm{CH}_{2}\right), 51.33,61.05\left(\mathrm{~N}-\mathrm{CH}_{2}\right)$, $100.06\left(\mathrm{~N}-\mathrm{CH}_{2}\right), 107.06\left(\mathrm{O}-\mathrm{CH}_{2}-\mathrm{O}\right), 108.34,117.20$, $121.33,123.54,125.85,146.15,146.94,167.64 \mathrm{ppm}$ $\left(\mathrm{CH}_{\text {arom, }} \mathrm{C}_{\text {arom, }} \mathrm{C}_{\text {butad }}\right) . \mathrm{MS}[+\mathrm{ESI}]: m / z 504[\mathrm{M}+\mathrm{Na}]^{+}$, $482[\mathrm{M}]^{+}, \mathrm{MS} / \mathrm{MS}[+\mathrm{ESI}]: \mathrm{m} / z 446[\mathrm{M}-\mathrm{Cl}]^{+}, 400$ $\left[\mathrm{M}-\left(\mathrm{Cl}+\mathrm{NO}_{2}\right)\right]^{+}, 365\left[\mathrm{M}-\left(2 \mathrm{Cl}+\mathrm{NO}_{2}\right)\right]^{+}, 332[\mathrm{M}-$ $\left.\left(3 \mathrm{Cl}+\mathrm{NO}_{2}\right)\right]^{+}, 309\left[\mathrm{M}-\left(3 \mathrm{Cl}+\mathrm{NO}_{2}+\mathrm{Na}+\mathrm{H}\right)\right]^{+}$. Micro analysis: $\mathrm{C}_{18} \mathrm{H}_{20} \mathrm{~N}_{3} \mathrm{O}_{4} \mathrm{~S}_{1} \mathrm{Cl}_{3}$ (M, 480.801). Calcd. C, 44.96; H, 4.19; N, 8.73; S, 6.66. Found C, 44.92; H, $4.14 ; \mathrm{N}, 8.74 ; \mathrm{S}, 6.61 \%$.

2.3e 3, 4, 4-Trichloro-2-nitro-(1E)-[propylsulphanyl1-(1-piperonylpiperazin-1-yl)]-1, 3-butadiene (7b): Compound $\mathbf{7 b}$ was synthesized from reaction of $\mathbf{4 b}$ $(1.0 \mathrm{~g}, 3.21 \mathrm{mmol})$ with 1-(piperonyl)piperazine $\mathbf{6 a}$ $(0.70 \mathrm{~g}, 3.21 \mathrm{mmol})$ according to method 3. Yield: $0.82 \mathrm{~g}(52 \%)$; Yellow oil. $R_{\mathrm{f}}: 0.60\left(\mathrm{CH}_{2} \mathrm{Cl}_{2}\right)$. IR
$\left(\mathrm{KBr}, \mathrm{cm}^{-1}\right): v=3051(\mathrm{Ar}-\mathrm{H}), 2963,2831,2814$, $2776(\mathrm{C}-\mathrm{H}), 1528,1656(\mathrm{C}=\mathrm{C}), 1274,1442(\mathrm{C}-$ $\mathrm{NO}_{2}$ ). ${ }^{1} \mathrm{H}-\mathrm{NMR}\left(499.74 \mathrm{MHz}, \mathrm{CDCl}_{3}\right): \delta=0.92(\mathrm{t}$, $\left.J=7.32 \mathrm{~Hz}, 3 \mathrm{H}, \mathrm{CH}_{3}\right), 1.5-1.7\left(\mathrm{~m}, 2 \mathrm{H}, \mathrm{S}-\mathrm{CH}_{2}-\right.$ $\mathrm{CH}_{2}$ ), 2.6 (sbr, 4H, $\mathrm{H}_{\text {piper }}$ ), 2.9 (t, $J=6.83 \mathrm{~Hz}, 2 \mathrm{H}$, $\left.\mathrm{S}-\mathrm{CH}_{2}\right), 3.4\left(\mathrm{~s}, 2 \mathrm{H}, \mathrm{N}-\mathrm{CH}_{2}\right), 3.6\left(\mathrm{sbr}, 4 \mathrm{H}, \mathrm{H}_{\text {piper }}\right.$ ), 5.87(s, 2H, O- $\left.\mathrm{CH}_{2}-\mathrm{O}\right), 6.6-6.9 \mathrm{ppm}\left(\mathrm{m}, 3 \mathrm{H}, \mathrm{H}_{\text {arom }}\right)$. ${ }^{13} \mathrm{C}-\mathrm{NMR}\left(125.66 \mathrm{MHz}, \mathrm{CDCl}_{3}\right): \delta=12.25\left(\mathrm{CH}_{3}\right)$, $22.22\left(\mathrm{~S}-\mathrm{CH}_{2}-\mathrm{CH}_{2}, \quad 36.32\left(\mathrm{~S}-\mathrm{CH}_{2}\right), \quad 51.18,60.92\right.$ $\left(\mathrm{N}-\mathrm{CH}_{2}\right), \quad 100.09\left(\mathrm{~N}-\mathrm{CH}_{2}\right), \quad 107.09\left(\mathrm{O}-\mathrm{CH}_{2}-\mathrm{O}\right)$, $108.47,116.98,121.55,123.55,125.89,146.26$, $146.94,168.10 \mathrm{ppm}\left(\mathrm{CH}_{\text {arom }}, \mathrm{C}_{\text {arom }}, \mathrm{C}_{\text {butad }}\right) . \mathrm{MS}$ [+ESI]: $m / z \quad 557 \quad[\mathrm{M}+\mathrm{Na}+\mathrm{K}]^{+}, \mathrm{MS} / \mathrm{MS}[+\mathrm{ESI}]: \mathrm{m} / z \quad 496$ $[\mathrm{M}+\mathrm{H}]^{+}, 459[\mathrm{M}-\mathrm{Cl}]^{+}, 413\left[\mathrm{M}-\left(\mathrm{Cl}+\mathrm{NO}_{2}\right)\right]^{+}, 346$ $\left[\mathrm{M}-\left(3 \mathrm{Cl}+\mathrm{NO}_{2}\right)\right]^{+}, \quad 323 \quad\left[\mathrm{M}-\left(3 \mathrm{Cl}+\mathrm{NO}_{2}+\mathrm{Na}+\mathrm{H}\right)\right]^{+}$. Micro analysis: $\mathrm{C}_{19} \mathrm{H}_{22} \mathrm{~N}_{3} \mathrm{O}_{4} \mathrm{~S}_{1} \mathrm{Cl}_{3}$ (M, 494.832). Calcd. C, 46.11; H, 4.48; N, 8.49; S, 6.47. Found C, 46.22; H, $4.44 ; \mathrm{N}, 8.54 ; \mathrm{S}, 6.51 \%$.

$2.3 \mathrm{f} 3,4,4-T r i c h l o r o-2-n i t r o-(1 E)$-[pentylsulphanyl4-(2-fluorophenylpiperazin-1-yl)]-1, 3-butadiene (7c): Compound $\mathbf{7 c}$ was synthesized from reaction of $\mathbf{4 d}$ $(0.5 \mathrm{~g}, 1.47 \mathrm{mmol})$ with 2-fluorophenylpiperazine $\mathbf{6 b}$ $(0.26 \mathrm{~g}, 1.47 \mathrm{mmol})$ according to method 3. Yield: $0.5 \mathrm{~g}$ (71\%); Yellow oil. $R_{\mathrm{f}}$ : $0.50\left(\mathrm{CHCl}_{3}\right)$. IR ( $\mathrm{KBr}$ pellet, $\left.\mathrm{cm}^{-1}\right): v=3045,3020(\mathrm{Ar}-\mathrm{H}), 2957,2928,2856$ $(\mathrm{C}-\mathrm{H}), 1529,1655(\mathrm{C}=\mathrm{C}), 1275,1501\left(\mathrm{C}-\mathrm{NO}_{2}\right) .{ }^{1} \mathrm{H}$ NMR $\left(499.74 \mathrm{MHz}, \mathrm{CDCl}_{3}\right): \delta=0.8(\mathrm{t}, J=6.83 \mathrm{~Hz}$, $\left.3 \mathrm{H}, \mathrm{CH}_{3}\right), 1.1-1.4\left(\mathrm{~m}, 4 \mathrm{H}, \mathrm{CH}_{2}\right), 1.6\left(\mathrm{~m}, 2 \mathrm{H}, \mathrm{S}-\mathrm{CH}_{2}-\right.$ $\left.\mathrm{CH}_{2}\right), 3.0\left(\mathrm{t}, J=7.32 \mathrm{~Hz}, 2 \mathrm{H}, \mathrm{S}-\mathrm{CH}_{2}\right), 3.2(\mathrm{~s}, \mathrm{br}$, $4 \mathrm{H}, \mathrm{H}_{\text {piper }}$ ), 3.8 (s, br, $\left.4 \mathrm{H}, \mathrm{H}_{\text {piper }}\right), 6.8-7.1 \mathrm{ppm}(\mathrm{m}, 4 \mathrm{H}$, $\left.\mathrm{H}_{\text {arom }}\right) .{ }^{13} \mathrm{C} \mathrm{NMR}\left(125.66 \mathrm{MHz}, \mathrm{CDCl}_{3}\right): \delta=12.81$ $\left(\mathrm{CH}_{3}\right), 21.11,28.47\left(\mathrm{CH}_{2}\right), 29.81\left(\mathrm{~S}-\mathrm{CH}_{2}-\mathrm{CH}_{2}\right)$, $34.55\left(\mathrm{~S}-\mathrm{CH}_{2}\right), 49.40,52.34\left(\mathrm{~N}-\mathrm{CH}_{2}\right), 115.44,122.92$, 122.98, 123.68, 123.70, 125.84, 137.37, 153.81, $155.78,168.39 \mathrm{ppm}\left(\mathrm{CH}_{\text {arom, }} \mathrm{C}_{\text {arom }}, \mathrm{C}_{\text {butad }}\right)$. Micro analysis: $\mathrm{C}_{19} \mathrm{H}_{23} \mathrm{O}_{2} \mathrm{~S}_{1} \mathrm{~N}_{3} \mathrm{Cl}_{3} \mathrm{~F}$ (M, 482.83). Calcd. C, 47.26; H, 4.80; N, 8.70; S, 6.64. Found: C, 47.89; N, 8.75; H, $4.67 ; \mathrm{S}, 6.02 \%$.

$2.3 \mathrm{~g}$ 3, 4, 4-Trichloro-2-nitro-(1E)-[pentylsulphanyl4-4-fluorophenylpiperazin-1-yl)]-1, 3-butadiene (7d): Compound 7d was synthesized from reaction of $\mathbf{4 d}$ $(0.5 \mathrm{~g}, 1.47 \mathrm{mmol})$ with 4-fluorophenylpiperazine $\mathbf{6 c}$ $(0.26 \mathrm{~g}, 1.47 \mathrm{mmol})$ according to method 3. Yield: $0.45 \mathrm{~g}(63 \%)$; Yellow oil. $R_{\mathrm{f}}$ : $0.50\left(\mathrm{CHCl}_{3}\right)$. IR $(\mathrm{KBr}$, pellet, $\left.\mathrm{cm}^{-1}\right): v=3055,3019(\mathrm{Ar}-\mathrm{H}), 2955,2932$, $2870(\mathrm{C}-\mathrm{H}), 1540,1654(\mathrm{C}=\mathrm{C}), 1271,1509\left(\mathrm{C}-\mathrm{NO}_{2}\right)$. ${ }^{1} \mathrm{H}$ NMR $\left(499.74 \mathrm{MHz}, \mathrm{CDCl}_{3}\right): \delta=0.85(\mathrm{t}, J=$ $\left.6.83 \mathrm{~Hz}, 3 \mathrm{H}, \mathrm{CH}_{3}\right), 1.1-1.4\left(\mathrm{~m}, 4 \mathrm{H}, \mathrm{CH}_{2}\right), 1.6(\mathrm{~m}, 2 \mathrm{H}$, $\left.\mathrm{S}-\mathrm{CH}_{2}-\mathrm{CH}_{2}\right), 2.9\left(\mathrm{t}, J=7.32 \mathrm{~Hz}, 2 \mathrm{H}, \mathrm{S}-\mathrm{CH}_{2}\right), 3.2$ (s, br, $4 \mathrm{H}, \mathrm{H}_{\text {piper }}$ ), 3.8 (s, br, $4 \mathrm{H}, \mathrm{H}_{\text {piper }}$ ), 6.8-7.1 ppm 
$\left(\mathrm{m}, 4 \mathrm{H}, \mathrm{H}_{\text {arom }}\right) \cdot{ }^{13} \mathrm{C}$ NMR $\left(125.66 \mathrm{MHz}, \mathrm{CDCl}_{3}\right): \delta=$ $12.81\left(\mathrm{CH}_{3}\right), 21.11,28.45\left(\mathrm{CH}_{2}\right), 29.79\left(\mathrm{~S}-\mathrm{CH}_{2}-\mathrm{CH}_{2}\right)$, $34.58\left(\mathrm{~S}-\mathrm{CH}_{2}\right), 49.78,51.81\left(\mathrm{~N}-\mathrm{CH}_{2}\right), 115.19,120.78$, $121.60,123.89,125.71,144.62,158.48,159.67$, $168.34 \mathrm{ppm}\left(\mathrm{CH}_{\text {arom, }} \mathrm{C}_{\text {arom, }} \mathrm{C}_{\text {butad }}\right)$. Micro analysis: $\mathrm{C}_{19} \mathrm{H}_{23} \mathrm{O}_{2} \mathrm{~S}_{1} \mathrm{~N}_{3} \mathrm{Cl}_{3} \mathrm{~F}$ (M, 482.83). Calcd. C, 47.26; H, $4.80 ; \mathrm{N}, 8.70 ; \mathrm{S}, 6.64$. Found: C, 47.38; H, 4.84; N, $8.90 ; \mathrm{S}, 6.62 \%$.

$2.3 \mathrm{~h}$ 3, 4, 4-Trichloro-2-nitro-(1E)-[pentylsulphanyl1-(4-diphenylmethyl)piperazin-1-yl)]-1, 3-butadiene (7e): Compound $7 \mathbf{e}$ was synthesized from reaction of $\mathbf{4 d}$ $(0.5 \mathrm{~g}, 1.47 \mathrm{mmol})$ with $N$-(diphenylmethyl)-piperazine 6d $(0.37 \mathrm{~g}, 1.47 \mathrm{mmol})$ according to method 3 . Yield $0.56 \mathrm{~g}(69 \%)$; Yellow oil. $R_{\mathrm{f}}\left(\mathrm{CHCl}_{3}\right): 0.38 ; \mathrm{IR}(\mathrm{KBr}$ pellet, $\left.\mathrm{cm}^{-1}\right): v=3060,3027(\mathrm{Ar}-\mathrm{H}), 2858,2928$ $(\mathrm{C}-\mathrm{H}), 1597,1660(\mathrm{C}=\mathrm{C}), 1286,1530\left(\mathrm{C}-\mathrm{NO}_{2}\right) ;{ }^{1} \mathrm{H}$ NMR $\left(499.74 \mathrm{MHz}, \mathrm{CDCl}_{3}\right): \delta 0.82(\mathrm{t}, J=6.83 \mathrm{~Hz}$, $\left.3 \mathrm{H}, \mathrm{CH}_{3}\right), 1.1-1.4\left(\mathrm{~m}, 4 \mathrm{H}, \mathrm{CH}_{2}\right), 1.5-1.6(\mathrm{~m}, 2 \mathrm{H}$, $\left.\mathrm{S}-\mathrm{CH}_{2}-\mathrm{CH}_{2}\right), 2.85\left(\mathrm{t}, j=7.32 \mathrm{~Hz}, 2 \mathrm{H}, \mathrm{S}-\mathrm{CH}_{2}\right.$ ), 2.5 (s, br, 4H, $\mathrm{H}_{\text {piper }}$ ), 3.55 (s, br, 4H, $\mathrm{H}_{\text {piper }}$ ), 4.4 $(\mathrm{s}, 1 \mathrm{H},-\mathrm{CH}<), 7.1-7.5 \mathrm{ppm}\left(\mathrm{m}, 10 \mathrm{H}, \mathrm{H}_{\text {arom. }}\right) ;{ }^{13} \mathrm{C}$ NMR $\left(125.66 \mathrm{MHz}, \mathrm{CDCl}_{3}\right): \delta 12.80\left(\mathrm{CH}_{3}\right), 21.08$, $28.44\left(\mathrm{CH}_{2}\right), 29.73\left(\mathrm{~S}-\mathrm{CH}_{2}-\mathrm{CH}_{2}\right), 34.44\left(\mathrm{~S}-\mathrm{CH}_{2}\right)$, $35.85, \quad 50.54 \quad\left(\mathrm{~N}-\mathrm{CH}_{2}\right), \quad 75.79 \quad(-\mathrm{CH}<), \quad 123.55$, $125.56,125.90,126.50,127.88,129.01,140.48$, $167.80 \mathrm{ppm}\left(\mathrm{CH}_{\text {arom, }} \mathrm{C}_{\text {arom, }} \mathrm{C}_{\text {butad }}\right)$. Micro analysis: $\mathrm{C}_{26} \mathrm{H}_{30} \mathrm{~N}_{3} \mathrm{O}_{2} \mathrm{~S}_{1} \mathrm{Cl}_{3}$ (M, 554.971). Calcd. C, 56.27; $\mathrm{H}$, 5.44; N, 7.57; S, 5.77. Found C, 56.47; H, 5.14; N, $7.59 ; \mathrm{S}, 5.70 \%$.

$2.3 \mathrm{i} 3,4,4-T r i c h l o r o-2-n i t r o-(1 E)$-[hexadecylsulphanyl1-(1-piperonylpiperazin-1-yl)]-1, 3-butadiene (7f): Compound 7f was synthesized from reaction of $\mathbf{4 f}$ $(0.5 \mathrm{~g}, 1.01 \mathrm{mmol})$ with 1-(piperonyl) piperazine $\mathbf{6 a}$ $(0.22 \mathrm{~g}, 1.01 \mathrm{mmol})$ according to method 3. Yield: $0.45 \mathrm{~g}(66 \%)$; m.p.: $188-189^{\circ} \mathrm{C} . R_{\mathrm{f}}: 0.55\left(\mathrm{CH}_{2} \mathrm{Cl}_{2}\right)$. IR $\left(\mathrm{KBr}, \mathrm{cm}^{-1}\right): v=3055(\mathrm{Ar}-\mathrm{H}), 2924,2853,2775$ $(\mathrm{C}-\mathrm{H}), 1530,1607 \quad(\mathrm{C}=\mathrm{C}), 1275,1490\left(\mathrm{C}-\mathrm{NO}_{2}\right)$. ${ }^{1} \mathrm{H}-\mathrm{NMR}\left(499.74 \mathrm{MHz}, \mathrm{CDCl}_{3}\right): \delta=0.79(\mathrm{t}, J=$ $\left.6.35 \mathrm{~Hz}, 3 \mathrm{H}, \mathrm{CH}_{3}\right), 1.0-1.4\left(\mathrm{~m}, 26 \mathrm{H}, \mathrm{CH}_{2}\right), 1.6(\mathrm{~m}$, $2 \mathrm{H}, \mathrm{S}-\mathrm{CH}_{2}-\mathrm{CH}_{2}$ ), 2.6 (sbr, $4 \mathrm{H}, \mathrm{H}_{\mathrm{piper}}$ ), 2.9 (t, $J=$ $\left.6.35 \mathrm{~Hz}, 2 \mathrm{H}, \mathrm{S}-\mathrm{CH}_{2}\right), 3.5\left(\mathrm{~s}, 2 \mathrm{H}, \mathrm{N}-\mathrm{CH}_{2}\right), 3.6(\mathrm{sbr}$, $\left.4 \mathrm{H}, \mathrm{H}_{\text {piper }}\right), \quad 5.9$ (s, 2H, O- $\left.\mathrm{CH}_{2}-\mathrm{O}\right), \quad 6.6-6.9 \mathrm{ppm}$ $\left(\mathrm{m}, 3 \mathrm{H}, \mathrm{H}_{\text {arom }}\right) .{ }^{13} \mathrm{C}-\mathrm{NMR}\left(125.66 \mathrm{MHz}, \mathrm{CDCl}_{3}\right)$ : $\delta=13.10\left(\mathrm{CH}_{3}\right), 21.67,27.52,27.66,28.02,28.22$, 28.33, 28.36, 28.50, 28.59, 28.63, 28.67, 28.68, 28.72, $30.90\left(\mathrm{CH}_{2}\right), 34.50\left(\mathrm{~S}-\mathrm{CH}_{2}-\mathrm{CH}_{2}\right), 38.25\left(\mathrm{~S}-\mathrm{CH}_{2}\right)$, $51.12, \quad 60.91\left(\mathrm{~N}-\mathrm{CH}_{2}\right), \quad 100.11\left(\mathrm{~N}-\mathrm{CH}_{2}\right), \quad 107.12$ $\left(\mathrm{O}-\mathrm{CH}_{2}-\mathrm{O}\right), 108.52,117.02,121.63,123.58,125.87$, 146.34, 146.97, $168.13 \mathrm{ppm}\left(\mathrm{CH}_{\text {arom, }} \mathrm{C}_{\text {arom, }} \mathrm{C}_{\text {butad }}\right)$. MS [+ESI]: $m / z 739[\mathrm{M}+\mathrm{Na}+\mathrm{K}]^{+}, 701[\mathrm{M}+\mathrm{Na}]^{+}$,
$678[\mathrm{M}]^{+}, \mathrm{MS} / \mathrm{MS}[+\mathrm{ESI}]: \mathrm{m} / z 642[\mathrm{M}-\mathrm{Cl}]^{+}, 596$ $\left[\mathrm{M}-\left(\mathrm{Cl}+\mathrm{NO}_{2}\right)\right]^{+}, 528\left[\mathrm{M}-\left(3 \mathrm{Cl}+\mathrm{NO}_{2}\right)\right]^{+}, 505[\mathrm{M}-$ $\left.\left(3 \mathrm{Cl}+\mathrm{NO}_{2}+\mathrm{Na}\right)\right]^{+}$. Micro analysis: $\mathrm{C}_{32} \mathrm{H}_{48} \mathrm{~N}_{3} \mathrm{O}_{4} \mathrm{~S}_{1} \mathrm{Cl}_{3}$ (M, 677.181). Calcd. C, 56.75; H, 7.14; N, 6.20; S, 4.73. Found C, 56.78; H, 7.24; N, 6.24; S, 4.75\%.

2.3j 1, 1'-Bis[3, 4, 4-trichloro-(1E)-(2-ethyl-hexyl-sulphanyl)2-nitro-1, 3-butadienyl]-homopiperazine (8h): Compound $8 \mathbf{h}$ was synthesized from reaction of $\mathbf{4 h}(0.5 \mathrm{~g}$, $1.31 \mathrm{mmol})$ with homopiperazine $(0.13 \mathrm{~g}, 1.31 \mathrm{mmol})$ according to method 2. Yield: $0.65 \mathrm{~g}(63 \%)$; Yellow oil. $R_{\mathrm{f}}\left(\mathrm{CH}_{2} \mathrm{Cl}_{2}\right): 0.48 ; \mathrm{IR}\left(\mathrm{KBr}, \mathrm{cm}^{-1}\right): v=2960,2929$, 2869, $2859(\mathrm{C}-\mathrm{H}), 1455,1672(\mathrm{C}=\mathrm{C}), 1277,1568$ $\left(\mathrm{C}-\mathrm{NO}_{2}\right) ;{ }^{1} \mathrm{H} \mathrm{NMR}\left(499.74 \mathrm{MHz}, \mathrm{CDCl}_{3}\right): \delta=0.8-0.9$ (t, $\left.J=6.83 \mathrm{~Hz}, 6 \mathrm{H}, 2 \mathrm{CH}_{3}\right), 1.1-1.4\left(\mathrm{~m}, 8 \mathrm{H}, 4 \mathrm{CH}_{2}\right)$, $1.55\left(\mathrm{~m}, 1 \mathrm{H}, \mathrm{S}-\mathrm{CH}_{2}-\mathrm{CH}\right), 3.0\left(\mathrm{~d}, 2 \mathrm{H}, \mathrm{S}-\mathrm{CH}_{2}\right), 2.2$ (s, br, $\mathrm{H}_{\text {homopiper }}$ ), $3.8 \mathrm{ppm}$ (s, br, $\mathrm{H}_{\text {homopiper }}$ ). ${ }^{13} \mathrm{C}$ NMR $\left(125.66 \mathrm{MHz}, \mathrm{CDCl}_{3}\right): \delta=12.68,12.99,13.10,13.18$ $\left(\mathrm{CH}_{3}\right), 21.67,21.79,24.47,27.57,27.77,28.17,28.63$, $28.68\left(\mathrm{CH}_{2}\right), 30.90,31.16\left(\mathrm{~S}-\mathrm{CH}_{2}-\mathrm{CH}\right), 38.52,39.40$ $\left(\mathrm{S}-\mathrm{CH}_{2}\right), 117.77,124.11,125.46,171.69 \mathrm{ppm}\left(\mathrm{C}_{\text {butad }}\right)$. MS [+ESI]: $m / z 790[\mathrm{M}]^{+}, 743\left[\mathrm{M}-\mathrm{NO}_{2}\right]^{+}$. Micro analysis: $\mathrm{C}_{29} \mathrm{H}_{44} \mathrm{~N}_{4} \mathrm{O}_{4} \mathrm{~S}_{2} \mathrm{Cl}_{6} \quad(M=789.545 \mathrm{~g} / \mathrm{mol})$. Calcd. C, 44.11; H, 5.61; N, 7.09; S, 8.12. Found C, 44.18; H, 5.65; N, 7.09; S, 8.16\%.

$2.3 \mathrm{k} \quad 3,4,4-T r i c h l o r o-2-n i t r o-(1 E)-[(2-e t h y l$-hexylsulphanyl)-1-homopiperazinyl]-1, 3-butadiene (9h): Compound $\mathbf{9 h}$ was synthesized from reaction of 4h $(0.5 \mathrm{~g}, 1.31 \mathrm{mmol})$ with homopiperazine $(0.13 \mathrm{~g}$, $1.31 \mathrm{mmol})$ according to method 3. Yield: $0.12 \mathrm{~g}$ (12\%); Yellow oil. $R_{\mathrm{f}}\left(\mathrm{CH}_{2} \mathrm{Cl}_{2}\right): 0.45$; IR(in $\mathrm{CHCl}_{3}$, $\left.\mathrm{cm}^{-1}\right): v=3849(\mathrm{~N}-\mathrm{H}), 2958,2919,2850(\mathrm{C}-\mathrm{H})$, 1486, $1696(\mathrm{C}=\mathrm{C}), 1278,1506\left(\mathrm{C}-\mathrm{NO}_{2}\right) ;{ }^{1} \mathrm{H}$ NMR $\left(499.74 \mathrm{MHz}, \mathrm{CDCl}_{3}\right): \delta=0.8(\mathrm{t}, J=7.32 \mathrm{~Hz}, 6 \mathrm{H}$, $\left.2 \mathrm{CH}_{3}\right), 1.0-1.4\left(\mathrm{~m}, 8 \mathrm{H}, 4 \mathrm{CH}_{2}\right), 1.5\left(\mathrm{~m}, 1 \mathrm{H}, \mathrm{S}-\mathrm{CH}_{2}-\right.$ $\mathrm{CH}$ ), 3.0 (d, 2H, S- $\mathrm{CH}_{2}$ ), 2.0 (s, br, $\mathrm{H}_{\text {homopiper }}$ ), 3.6 (s, br, $\mathrm{H}_{\text {homopiper }}$ ), $5.3 \mathrm{ppm}(\mathrm{s}, 1 \mathrm{H}, \mathrm{NH}) .{ }^{13} \mathrm{C}$ NMR $\left(125.66 \mathrm{MHz}, \mathrm{CDCl}_{3}\right): \delta=12.97,13.63\left(\mathrm{CH}_{3}\right), 21.80$, 24.46, 27.55, $28.68\left(\mathrm{CH}_{2}\right), 31.15\left(\mathrm{~S}-\mathrm{CH}_{2}-\mathrm{CH}\right), 38.59$ $\left(\mathrm{S}-\mathrm{CH}_{2}\right), 108.74,131.55,154.84,178.34 \mathrm{ppm}\left(\mathrm{C}_{\text {butad }}\right)$. MS [+ESI]: $m / z 540[\mathrm{M}+4 \mathrm{Na}+3 \mathrm{H}]^{+}$. Micro analysis: $\mathrm{C}_{17} \mathrm{H}_{28} \mathrm{~N}_{3} \mathrm{O}_{2} \mathrm{~S}_{1} \mathrm{Cl}_{3}(M=444.855 \mathrm{~g} / \mathrm{mol})$. Calcd. C, 45.90; H, 6.34; N, 9.44; S, 7.20. Found C, 45.92; H, $6.38 ; \mathrm{N}, 9.49 ; \mathrm{S}, 7.26 \%$.

2.31 3, 4, 4-Trichloro-2-nitro-1-bis(11-sulphanyl-1undecanol)-1, 3-butadiene (10e): Compound 10e was synthesized from reaction of $\mathbf{1}(2 \mathrm{~g}, 7.37 \mathrm{mmol})$ with 11-mercapto-1-undecanol 3e (4.51 g, $22.06 \mathrm{mmol})$ according to method 2. Yield: $1.3 \mathrm{~g}(50 \%)$; Yellow oil. $R_{\mathrm{f}}$ : $0.50\left(\mathrm{CHCl}_{3}\right)$. IR $\left(\mathrm{KBr}\right.$ pellet, $\left.\mathrm{cm}^{-1}\right)$ : 
$v=3338(\mathrm{OH}), 2926,2853(\mathrm{C}-\mathrm{H}), 1535,1574(\mathrm{C}=\mathrm{C})$, 1292, $1464\left(\mathrm{C}-\mathrm{NO}_{2}\right),{ }^{1} \mathrm{H}$ NMR $\left(499.74 \mathrm{MHz}, \mathrm{CDCl}_{3}\right)$ : $\delta=1.1-1.4\left(\mathrm{~m}, 28 \mathrm{H}, \mathrm{CH}_{2}\right), 1.45-1.5\left(\mathrm{~m}, 4 \mathrm{H}, \mathrm{S}-\mathrm{CH}_{2}-\right.$ $\left.\mathrm{CH}_{2}\right), 1.55-1.7\left(\mathrm{~m}, 4 \mathrm{H}, \mathrm{HO}-\mathrm{CH}_{2}-\mathrm{CH}_{2}\right), 3.0(\mathrm{~s}, 2 \mathrm{H}$, $\mathrm{OH}), 3.6\left(\mathrm{t}, J=6.84 \mathrm{~Hz}, 4 \mathrm{H}, \mathrm{S}-\mathrm{CH}_{2}\right), 4.2 \mathrm{ppm}(\mathrm{m}$, $\left.4 \mathrm{H}, \mathrm{HO}-\mathrm{CH}_{2}\right) \cdot{ }^{13} \mathrm{C}$ NMR $\left(125.66 \mathrm{MHz}, \mathrm{CDCl}_{3}\right): \delta=$ 23.62, 24.72, 25.85, 27.35, 27.50, 27.64, $27.67\left(\mathrm{CH}_{2}\right)$, 28.02, $28.04\left(\mathrm{~S}-\mathrm{CH}_{2}-\mathrm{CH}_{2}\right), 28.35,28.38\left(\mathrm{HO}-\mathrm{CH}_{2}-\right.$ $\left.\mathrm{CH}_{2}\right), 28.43,28.45\left(\mathrm{~S}-\mathrm{CH}_{2}\right), 62.11,62.14\left(\mathrm{HO}-\mathrm{CH}_{2}\right)$, $119.28,122.13,126.64,161.99 \mathrm{ppm}\left(\mathrm{C}_{\text {butad }}\right) . \quad \mathrm{MS}$ [+ESI]: $m / z 608[\mathrm{M}]^{+}, 562\left[\mathrm{M}-\mathrm{NO}_{2}\right]^{+}$; Micro analysis: $\mathrm{C}_{26} \mathrm{H}_{46} \mathrm{O}_{4} \mathrm{~S}_{2} \mathrm{~N}_{1} \mathrm{Cl}_{3}$ (M, 607.149). Calcd. C, 51.43; H, 7.63; N, 2.30; S, 10.56. Found C, 51.42; H, 7.67; N, $2.32 ; \mathrm{S}, 10.59 \%$.

$2.3 \mathrm{~m}$ 3, 4-Dichloro-2-nitro-1, 1, 4-tris(propylsulphanyl)1, 3-butadiene $(\mathbf{1 1 b})$ : Compound $\mathbf{1 1 b}$ was synthesized from reaction of $\mathbf{1}(2 \mathrm{~g}, 7.37 \mathrm{mmol})$ with propanethiol $\mathbf{3 b}(1.68 \mathrm{~g}, 22.11 \mathrm{mmol})$ according to method 2. Yield $1.9 \mathrm{~g}(66 \%)$; Yellow oil. $R_{\mathrm{f}}$ : (petroleum ether- $\left.\mathrm{CCl}_{4}(1: 1)\right]$ : $0.32 ; \mathrm{IR}\left(\mathrm{KBr}, \mathrm{cm}^{-1}\right)$ : $v=2990(\mathrm{C}-\mathrm{H}), 1560,1630 \quad(\mathrm{C}=\mathrm{C}), 1280,1530$ $\left(\mathrm{C}-\mathrm{NO}_{2}\right) ;{ }^{1} \mathrm{H}-\mathrm{NMR}\left(499.74 \mathrm{MHz}, \mathrm{CDCl}_{3}\right): \delta 0.95(\mathrm{t}$, $\left.J=7.32 \mathrm{~Hz}, 3 \mathrm{H}, \mathrm{CH}_{3}\right), 1.60\left(\mathrm{~m}, 2 \mathrm{H}, \mathrm{S}-\mathrm{CH}_{2}-\right.$ $\left.\mathrm{CH}_{2}\right), 2.95 \mathrm{ppm}$ (t, $J=7.32 \mathrm{~Hz}, 2 \mathrm{H}, \mathrm{S}-\mathrm{CH}_{2}$ ); ${ }^{13} \mathrm{C}-\mathrm{NMR}\left(125.66 \mathrm{MHz}, \mathrm{CDCl}_{3}\right): \delta 12.38\left(\mathrm{CH}_{3}\right), 22.44$ $\left(\mathrm{S}-\mathrm{CH}_{2}-\mathrm{CH}_{2}\right), 37.41\left(\mathrm{~S}-\mathrm{CH}_{2}\right), 123.64,127.99,142.78$, $152.55 \mathrm{ppm}\left(\mathrm{C}_{\text {butad }}\right)$; $\mathrm{MS}[+\mathrm{ESI}]: \mathrm{m} / z \quad 390.24[\mathrm{M}]^{+}$; Micro analysis: $\mathrm{C}_{13} \mathrm{H}_{21} \mathrm{~N}_{1} \mathrm{O}_{2} \mathrm{~S}_{3} \mathrm{Cl}_{2}(\mathrm{M}, 390.41 \mathrm{~g} / \mathrm{mol})$. Calcd. C, 39.99; H, 5.42; N, 3.58; S, 24.63. Found C, $40.25 ; \mathrm{H}, 5.75 ; \mathrm{N}, 4.00 ; \mathrm{S}, 23.98 \%$.

2.3n 4, 4-Trichloro-2-nitro-1-tris(11-sulphanyl-1undecanol)-1, 3-butadiene (11e): Compound 11e was synthesized from reaction of $\mathbf{1}(2 \mathrm{~g}, 7.37 \mathrm{mmol})$ with 11-mercapto-1-undecanol 3e (4.51 g, $22.06 \mathrm{mmol})$ according to method 2. Yield: $0.5 \mathrm{~g}$ (35\%); Yellow oil. $R_{\mathrm{f}}: 0.40\left(\mathrm{CHCl}_{3}\right)$. IR $\left(\mathrm{KBr}\right.$ pellet, $\left.\mathrm{cm}^{-1}\right): v=$ 3362(OH), 2924, $2851(\mathrm{C}-\mathrm{H}), 1527,1550(\mathrm{C}=\mathrm{C})$, 1287, $1469\left(\mathrm{C}-\mathrm{NO}_{2}\right) .{ }^{1} \mathrm{H}$ NMR $\left(499.74 \mathrm{MHz}, \mathrm{CDCl}_{3}\right)$ : $\delta=1.1-1.4\left(\mathrm{~m}, 35 \mathrm{H}, \mathrm{CH}_{2}\right), 1.43-1.5\left(\mathrm{~m}, 6 \mathrm{H}, \mathrm{S}-\mathrm{CH}_{2}-\right.$ $\left.\mathrm{CH}_{2}\right), 1.6-1.7\left(\mathrm{~m}, 6 \mathrm{H}, \mathrm{HO}-\mathrm{CH}_{2}-\mathrm{CH}_{2}\right), 3.1(\mathrm{~s}, 3 \mathrm{H}$, $\mathrm{OH}), 3.6\left(\mathrm{t}, J=6.84 \mathrm{~Hz}, 6 \mathrm{H}, \mathrm{S}-\mathrm{CH}_{2}\right), 4.3 \mathrm{ppm}(\mathrm{m}$, $\left.6 \mathrm{H}, \mathrm{HO}-\mathrm{CH}_{2}\right) .{ }^{13} \mathrm{C}$ NMR $\left(125.66 \mathrm{MHz}, \mathrm{CDCl}_{3}\right): \delta=$ $23.11,23.15,23.24,23.62,23.65,24.72,25.85,27.35$, 27.50, 27.64, $27.67\left(\mathrm{CH}_{2}\right), 28.12,28.14\left(\mathrm{~S}-\mathrm{CH}_{2}-\right.$ $\left.\mathrm{CH}_{2}\right), 28.45,28.48\left(\mathrm{HO}-\mathrm{CH}_{2}-\mathrm{CH}_{2}\right), 28.43,28.45$, $28.46\left(\mathrm{~S}-\mathrm{CH}_{2}\right), 62.11,62.15,62.20\left(\mathrm{HO}-\mathrm{CH}_{2}\right), 117.28$ $123.13,155.64,162.99 \mathrm{ppm}\left(\mathrm{C}_{\text {butad }}\right)$. MS [-ESI]: $\mathrm{m} / z$ $758[\mathrm{M}-\mathrm{OH}]^{-}$; Micro analysis: $\mathrm{C}_{37} \mathrm{H}_{69} \mathrm{O}_{5} \mathrm{~S}_{3} \mathrm{~N}_{1} \mathrm{Cl}_{2}(\mathrm{M}$, 775.064). Calcd. C, 57.33; H, 8.97; N, 1.80; S, 12.41. Found C, 57.32; H, 8.99; N, 1.82; S, $12.38 \%$. 2.3o 3, 4-Dichloro-2-nitro-1, 1, 3-tris(octadecylsulphanyl)1,3-butadiene (11g): Compound $\mathbf{1 1 g}$ was synthesized from reaction of $\mathbf{1}(2 \mathrm{~g}, 7.37 \mathrm{mmol})$ with octadecanethiol $\mathbf{3 g}(6.33 \mathrm{~g}, 22.11 \mathrm{mmol})$ according to method

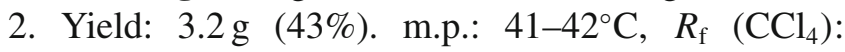
0.54; IR $\left(\mathrm{KBr}, \mathrm{cm}^{-1}\right): v=2980,2895,2820(\mathrm{C}-\mathrm{H})$, 1580, $1630(\mathrm{C}=\mathrm{C}), 1280,1530\left(\mathrm{C}-\mathrm{NO}_{2}\right) ;{ }^{1} \mathrm{H}$ NMR $\left(499.74 \mathrm{MHz}, \mathrm{CDCl}_{3}\right): \delta 0.88(\mathrm{t}, J=7.32 \mathrm{~Hz}, 3 \mathrm{H}$, $\left.\mathrm{CH}_{3}\right), 1.2-1.5\left(\mathrm{~m}, 30 \mathrm{H}, \mathrm{CH}_{2}\right), 1.60\left(\mathrm{~m}, 2 \mathrm{H}, \mathrm{S}-\mathrm{CH}_{2}-\right.$ $\left.\mathrm{CH}_{2}\right), 2.95 \mathrm{ppm}\left(\mathrm{t}, J=7.32 \mathrm{~Hz}, 2 \mathrm{H}, \mathrm{S}-\mathrm{CH}_{2}\right):{ }^{13} \mathrm{C}$ NMR $\left(125.66 \mathrm{MHz}, \mathrm{CDCl}_{3}\right): \delta 13.08\left(\mathrm{CH}_{3}\right), 21.67$, $27.67,27.81,27.83,28.10,28.13,28.15,28.26,28.35$, 28.43, 28.46, 28.57, 28.64, 28.65, $28.74\left(\mathrm{CH}_{2}\right), 30.92$ $\left(\mathrm{S}-\mathrm{CH}_{2}-\mathrm{CH}_{2}\right), 35.57\left(\mathrm{~S}-\mathrm{CH}_{2}\right), 123.26,128.14,142.53$, $152.84 \mathrm{ppm}\left(\mathrm{C}_{\text {butad }}\right)$; $\mathrm{MS}$ (+ESI): $m / z 1021.81[\mathrm{M}]^{+}$; $975.84[\mathrm{M}-46]^{+}$; Micro analysis: $\mathrm{C}_{58} \mathrm{H}_{111} \mathrm{~N}_{1} \mathrm{O}_{2} \mathrm{~S}_{3} \mathrm{Cl}_{2}$ (M, 1021.63). Calcd. C, 68.18; H, 10.95; N, 1.37; S, 9.41. Found C, 68.25; H, 11.22; N, 1.29; S, 9.28\%.

2.3p 1,3,4-Trichloro-4-bromo-2-nitro-(1E)-(2-ethylhexyl-sulphanyl)-1, 3-butadiene $(\mathbf{1 2 h})$ : Compound $\mathbf{1 2 h}$ was synthesized from reaction of $2(2 \mathrm{~g}, 6.33 \mathrm{mmol})$ with 2-ethyl-hexanethiol $\mathbf{3 h} \quad(0.92 \mathrm{~g}, \quad 6.33 \mathrm{mmol})$ according to method 1. Yield: $1.75 \mathrm{~g}(65 \%)$; Yellow oil. $R_{\mathrm{f}}\left(\mathrm{CCl}_{4}\right): 0.38 ; \mathrm{IR}\left(\mathrm{KBr}, \mathrm{cm}^{-1}\right): v=2960,2929$, 2872, $2859(\mathrm{C}-\mathrm{H}), 1459,1605(\mathrm{C}=\mathrm{C}), 1312,1533$ $\left(\mathrm{C}-\mathrm{NO}_{2}\right) ;{ }^{1} \mathrm{H} \mathrm{NMR}\left(499.74 \mathrm{MHz}, \mathrm{CDCl}_{3}\right): \delta=0.8-0.9$ $\left(\mathrm{t}, J=6.83 \mathrm{~Hz}, 6 \mathrm{H}, 2 \mathrm{CH}_{3}\right), 1.1-1.4\left(\mathrm{~m}, 8 \mathrm{H}, 4 \mathrm{CH}_{2}\right)$, $1.6\left(\mathrm{~m}, 1 \mathrm{H}, \mathrm{S}-\mathrm{CH}_{2}-\mathrm{CH}\right), 3.1 \mathrm{ppm}\left(\mathrm{d}, 2 \mathrm{H}, \mathrm{S}-\mathrm{CH}_{2}\right) .{ }^{13} \mathrm{C}$ NMR $\left(125.66 \mathrm{MHz}, \mathrm{CDCl}_{3}\right): \delta=12.99,13.01\left(\mathrm{CH}_{3}\right)$, 21.82, 24.61, 24.65, $27.25\left(\mathrm{CH}_{2}\right), 31.21\left(\mathrm{~S}-\mathrm{CH}_{2}-\mathrm{CH}\right)$, $38.52\left(\mathrm{~S}-\mathrm{CH}_{2}\right), 122.11,124.09,157.10,187.81 \mathrm{ppm}$ $\left(\mathrm{C}_{\text {butad }}\right)$. Micro analysis: $\mathrm{C}_{12} \mathrm{H}_{17} \mathrm{~N}_{1} \mathrm{O}_{2} \mathrm{~S}_{1} \mathrm{Cl}_{3} \mathrm{Br}_{1}(M=$ 425.602 g/mol). Calcd. C, 33.86; H, 4.02; N, 3.29; S, 7.53. Found C, 33.89; H, 3.05; N, 3.24; S, 7.55\%.

$2.3 q$ 3, 4-Dichloro-4-bromo-2-nitro-1-(E)-(2-ethlyhexyl-sulphanyl)-1-homopiperazinyl-1, 3-butadiene (13h): Compound $13 \mathrm{~h}$ was synthesized from reaction of 12h $(0.5 \mathrm{~g}, 1.17 \mathrm{mmol})$ with homopiperazine $(0.11 \mathrm{~g}$, $1.17 \mathrm{mmol}$ ) according to method 1. Yield: $0.18 \mathrm{~g}$ (17\%); Yellow oil. $R_{\mathrm{f}}\left(\mathrm{CHCl}_{3}\right)$ : 0.38; $\mathrm{IR}\left(\right.$ in $\mathrm{CHCl}_{3}$, $\left.\mathrm{cm}^{-1}\right): v=3784(\mathrm{~N}-\mathrm{H}), 2957,2922,2851(\mathrm{C}-\mathrm{H})$, 1429, $1667(\mathrm{C}=\mathrm{C}), 1276,1509\left(\mathrm{C}-\mathrm{NO}_{2}\right) ;{ }^{1} \mathrm{H}$ NMR (499.74 MHz, $\left.\mathrm{CDCl}_{3}\right): \delta=0.8(\mathrm{t}, J=7.32 \mathrm{~Hz}, 6 \mathrm{H}$, $\left.2 \mathrm{CH}_{3}\right), 1.0-1.4\left(\mathrm{~m}, 8 \mathrm{H}, 4 \mathrm{CH}_{2}\right), 1.6\left(\mathrm{~m}, 1 \mathrm{H}, \mathrm{S}-\mathrm{CH}_{2}-\right.$ $\mathrm{CH}), 3.1\left(\mathrm{~d}, 2 \mathrm{H}, \mathrm{S}-\mathrm{CH}_{2}\right), 2.0$ (s, br, $\left.\mathrm{H}_{\text {homopiper }}\right), 3.6$ (t, $\left.J=7.32, \mathrm{H}_{\text {homopiper }}\right), 5.2 \mathrm{ppm}(\mathrm{s}, 1 \mathrm{H}, \mathrm{NH}) .{ }^{13} \mathrm{C} \mathrm{NMR}$ $\left(125.66 \mathrm{MHz}, \mathrm{CDCl}_{3}\right): \delta=12.98,13.09\left(\mathrm{CH}_{3}\right), 21.67$, 21.80, 27.57, $28.34\left(\mathrm{CH}_{2}\right), 31.20\left(\mathrm{~S}-\mathrm{CH}_{2}-\mathrm{CH}\right), 38.07$ $\left(\mathrm{S}-\mathrm{CH}_{2}\right), 126.40,134.55,155.74,179.03 \mathrm{ppm}\left(\mathrm{C}_{\text {butad }}\right)$. MS [+ESI]: $m / z 537[\mathrm{M}+\mathrm{Na}+2 \mathrm{H}]^{+}$. Micro analysis: 
$\mathrm{C}_{17} \mathrm{H}_{28} \mathrm{~N}_{3} \mathrm{O}_{2} \mathrm{~S}_{1} \mathrm{Cl}_{2} \mathrm{Br}_{1}(M=489.306 \mathrm{~g} / \mathrm{mol})$. Calcd. C, 41.73; H, 5.76; N, 8.58; S, 6.55. Found C, 41.72; H, $5.75 ; \mathrm{N}, 8.54 ; \mathrm{S}, 6.56 \%$.

2.3r 1, I'-Bis[3, 4-dichloro-4-bromo-1(E)-(2-ethyl-hexylsulphanyl)-2-nitro-1, 3-butadienyl] homopiperazine (14h): Compound 14h was synthesized from reaction of 12h $(0.5 \mathrm{~g}, 1.17 \mathrm{mmol})$ with homopiperazine $(0.11 \mathrm{~g}$, $1.17 \mathrm{mmol})$ according to method 1 . Yield: $0.65 \mathrm{~g}$ (63\%), Yellow oil. $R_{\mathrm{f}}\left(\mathrm{CHCl}_{3}\right): 0.48 ; \mathrm{IR}\left(\mathrm{KBr}, \mathrm{cm}^{-1}\right)$ : $v=2957,2922,2850(\mathrm{C}-\mathrm{H}), 1463,1647(\mathrm{C}=\mathrm{C})$, 1270, $1510\left(\mathrm{C}-\mathrm{NO}_{2}\right) ;{ }^{1} \mathrm{H}$ NMR $\left(499.74 \mathrm{MHz}, \mathrm{CDCl}_{3}\right)$ : $\delta=0.7-0.9\left(\mathrm{t}, J=6.83 \mathrm{~Hz}, 12 \mathrm{H}, 4 \mathrm{CH}_{3}\right), 1.1-1.4(\mathrm{~m}$, $\left.16 \mathrm{H}, 8 \mathrm{CH}_{2}\right), 1.6\left(\mathrm{~m}, 2 \mathrm{H}, \mathrm{S}-\mathrm{CH}_{2}-\mathrm{CH}\right), 3.0(\mathrm{~d}, 4 \mathrm{H}, \mathrm{S}-$ $\mathrm{CH}_{2}$ ), 2.2 (s, br, $\left.\mathrm{H}_{\text {homopiper }}\right), 3.8 \mathrm{ppm}\left(\mathrm{m}, \mathrm{H}_{\text {homopiper }}\right) .{ }^{13} \mathrm{C}$ NMR $\left(125.66 \mathrm{MHz}, \mathrm{CDCl}_{3}\right): \delta=12.99,13.01,13.10$, $13.39\left(\mathrm{CH}_{3}\right), 21.67,21.79,24.49,26.07,27.58,28.34$, 28.64, $28.68\left(\mathrm{CH}_{2}\right), 30.91,31.18\left(\mathrm{~S}-\mathrm{CH}_{2}-\mathrm{CH}\right), 38.52$, $39.34\left(\mathrm{~S}-\mathrm{CH}_{2}\right), 113.04,126.93,128.83,168.27 \mathrm{ppm}$ $\left(\mathrm{C}_{\text {butad }}\right)$. MS [+ESI]: $m / z 900[\mathrm{M}+\mathrm{Na}]^{+}$, Micro analysis: $\mathrm{C}_{29} \mathrm{H}_{44} \mathrm{~N}_{4} \mathrm{O}_{4} \mathrm{~S}_{2} \mathrm{Cl}_{4} \mathrm{Br}_{2}(M=878.44 \mathrm{~g} / \mathrm{mol})$. Calcd. C, 39.65; H, 5.04; N, 6.37; S, 7.30. Found C, 39.68; H, $5.05 ; \mathrm{N}, 6.39 ; \mathrm{S}, 7.36 \%$.

2.3s 3,4-Dichloro-4-bromo-2-nitro-(1E)-[cyclo-(2butylaminoethylsulphanyl)]-1, 3-butadiene (15): Compound 15 was synthesized from reaction of 2 ( $1 \mathrm{~g}$, $3.16 \mathrm{mmol})$ with 2-(butylamino)ethanethiol $\mathbf{3 i}(0.42 \mathrm{~g}$, $3.16 \mathrm{mmol})$ according to method 1 . Yield: $0.9 \mathrm{~g}(76 \%)$. Yellow solid. m.p.: $145-146^{\circ} \mathrm{C} . R_{\mathrm{f}}: 0.50\left(\mathrm{CHCl}_{3}\right)$. IR $\left(\mathrm{KBr}, \mathrm{cm}^{-1}\right): v=2951,2932,2869(\mathrm{C}-\mathrm{H}), 1530,1581$ $(\mathrm{C}=\mathrm{C}), 1255,1456\left(\mathrm{C}-\mathrm{NO}_{2}\right) .{ }^{1} \mathrm{H}-\mathrm{NMR}(499.74 \mathrm{MHz}$, $\left.\mathrm{CDCl}_{3}\right): \delta=0.9\left(\mathrm{t}, J=7.32 \mathrm{~Hz}, 3 \mathrm{H}, \mathrm{CH}_{3}\right), 1.5$ $\left(\mathrm{m}, 4 \mathrm{H}, \mathrm{CH}_{2}\right), 3.5\left(\mathrm{t}, J=6.84 \mathrm{~Hz}, \mathrm{~S}-\mathrm{CH}_{2}\right), 3.8-$ $4.1 \mathrm{ppm}\left(\mathrm{m}, 4 \mathrm{H}, \mathrm{N}-\mathrm{CH}_{2}\right) .{ }^{13} \mathrm{C}-\mathrm{NMR}(125.66 \mathrm{MHz}$, $\left.\mathrm{CDCl}_{3}\right): \delta=12.68\left(\mathrm{CH}_{3}\right), 19.10\left(\mathrm{CH}_{2}\right), 26.22(\mathrm{~N}-$ $\left.\mathrm{CH}_{2}-\mathrm{CH}_{2}\right), 28.87\left(\mathrm{~S}-\mathrm{CH}_{2}\right), 50.85,56.36\left(\mathrm{~N}-\mathrm{CH}_{2}\right)$, $112.54,124.47,126.38,167.03 \mathrm{ppm}\left(\mathrm{C}_{\text {butad }}\right)$. MS [+ESI]: $m / z 377[\mathrm{M}]^{+}, 399[\mathrm{M}+\mathrm{Na}]^{+}$. Micro analysis: $\mathrm{C}_{10} \mathrm{H}_{13} \mathrm{O}_{2} \mathrm{~S}_{1} \mathrm{~N}_{2} \mathrm{Cl}_{2} \mathrm{Br}_{1}$ (M, 376.102). Calcd. C, 31.93; H, 3.48; N, 7.44; S, 8.52. Found C, 31.92; H, 3.47; N, $7.42 ; \mathrm{S}, 8.58 \%$.

\section{Results and discussion}

\subsection{Chemistry}

These electronically remarkable molecules can be used for a broad variety of synthetic applications since the reactions can be carried out by various methods ranging from nucleophilic vinylic substitution $\left(\mathrm{S}_{\mathrm{N}}\right.$ Vinylic) pathway via the addition-elimination route and ring cleavage to addition reactions of the trichlorovinyl group activated by the nitro group which also provides regioselectivity to molecules.

Nitrodiene compounds $\mathbf{1}$ and $\mathbf{2}$ were obtained from by direct nitration of the corresponding dienes. 1, 1 , 3, 4, 4-pentachloro-2-nitro-1, 3-butadiene 1 was gained from the nitration of $1,1,3,4,4$-pentachloro-2- $\mathrm{H}$ 1, 3-butadiene with concentrated $\mathrm{HNO}_{3}$ in about $40 \%$ yield. Nitration of a mixture of two geometric isomers of 1, 1, 3, 4-tetrachloro-4-bromo-2-nitro-1, 3-butadiene 2 with concentrated $\mathrm{HNO}_{3}$ occurs at the dichlorovinyl group by electrophilic substitution of the $\mathrm{H}$ atom by the nitro group with the formation of a mixture of $E$ - and $Z$-isomers of 1, 1, 3, 4-tetrachloro-4-bromo-2-nitro-1, 3 -butadiene in $\sim 40 \%$ yield. $^{23,24}$

The reaction of pentachloro-2-nitro-1, 3-butadiene 1 with one molar equivalent of ethanethiol 3a, propanethiol 3b, pentanethiol 3d, hexadecanthiol 3f, 2ethyl-hexanethiol $\mathbf{3 h}$ in dichloromethane at room temperature furnishes $(E)$-1, 3, 4, 4-tetrachloro-2-nitro-1(alkylsulphanyl)-1, 3-butadiene compounds $\mathbf{4 a},{ }^{14} \mathbf{4 b},{ }^{25}$ $\mathbf{4 d}, \mathbf{4 f},{ }^{14} \mathbf{4 h}$ as a single isomer $(75 \%$ yield for $\mathbf{4 d}$ and $66 \%$ for $4 \mathbf{h})$. (E)-4-bromo-1, 3, 4-trichloro-2-nitro-1(alkylsulphanyl)-1, 3-butadiene was synthesized from reaction of 4-bromo-1, 1, 3, 4-tetrachloro-2-nitrobuta1, 3-diene 12h (63\% yield) with 2-ethyl-hexanethiol $\mathbf{3 h}$ according to same method. These known (4a, $\mathbf{4 b}$, 4f) and unknown (4d, $\mathbf{4 h}, \mathbf{1 2 h})$ mono(thio)substituted nitrobutadiene compounds were used as the starting compounds with various nuchleophilic compounds at room temperature. It is expected that mono(thio)substituted nitrobutadienes yielded products such as a mixture of $E$ - and $Z$-isomers. However, in the crystal structure of $\mathrm{S}$ - and $\mathrm{N}, \mathrm{S}$-substituted nitrodienes reported in previous studies, it was noted that mono(thio)substituted nitrobutadienes were $E$-isomers and N, S-substituted nitrobutadienes were also $E$ isomers. As a consequence, it might be thought that the derived new nitrodiene products were probably $E$ isomers. ${ }^{13,17,25-27}$ The reactions of thiomorpholine and piperazine derivatives with S-substituted-tetrachloro-2nitro-1, 3-butadiene $(\mathbf{4 a}, \mathbf{4 b}, \mathbf{4 d}, \mathbf{4 f})$ gave the novel N, S-substituted-trichloro-2-nitro-1, 3-butadiene compounds (5d, 7a-f). These substitution reactions proceed by addition-elimination reaction mechanism (scheme 1). First, an addition of the attacking reagent to the C,C double bond occurs, and in a second step the intermediate product is stabilized by elimination of hydrogen chloride.

Compounds $8 \mathrm{~h}, 9 \mathrm{~h}, \mathbf{1 3 h}$ and $14 \mathrm{~h}$ were obtained from the reactions of $\mathbf{4 h}$ and $\mathbf{1 2 h}$ with homopiperazine. Disubstituted butadineyl homopiperazine compounds 8h and 14h are interesting unsaturated compounds 


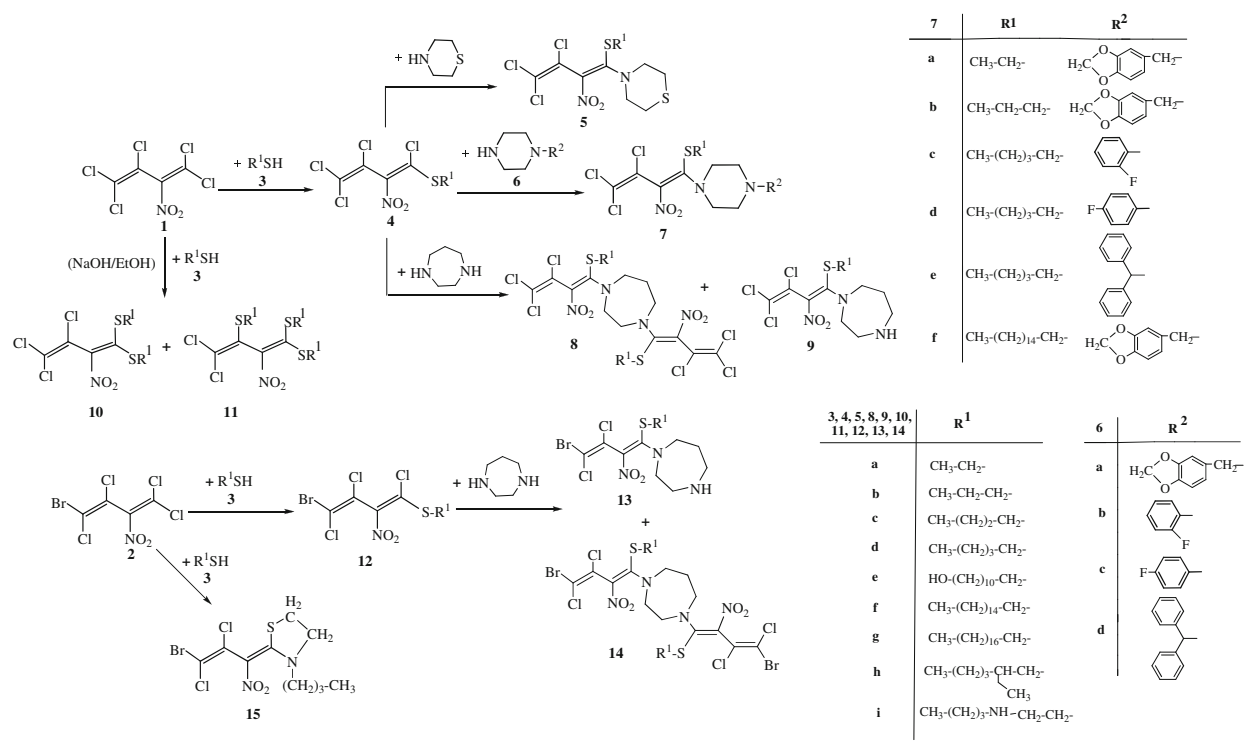

Scheme 1. Synthesis pathway of polyhalo-2-nitro-1, 3-butadiene compounds.

(scheme 1). Disubstituted homopiperazine compound $\mathbf{8 c}$ was earlier obtained from the reaction of mono(thio)substituted diene $\mathbf{4 c}$ with homopiperazine. ${ }^{18}$ Crystal structure of compound $\mathbf{8 c}$ was determined in this study.

It is well-known that the reactions of 2-nitrodiene with some thiols follow a classical $\mathrm{S}_{\mathrm{N}}$ Vin process. ${ }^{28}$ The 2-nitrodiene initially reacts at the terminal C-1 carbon atom to yield the product of mono(thio)substituted2-nitrobutadienes or 1, 1-bis(thio)substituted-2-nitrobutadienes. Under more rigid conditions, a subsequent substitution of chlorine atom on C-3 carbon atom is possible. Use of three equivalents of propanthiol $\mathbf{3 b}$, 11-mercapto-1-undecanol $\mathbf{3 e}$ and octadecanthiol $\mathbf{3 g}$ in the presence of sodium hydroxide in ethanol at room temperature with 1, 1, 3, 4, 4-pentachloro-2-nitro1, 3-butadiene 1 provides the di- and trisubstituted polychloro-2-nitro-1, 3-butadienes $10 e, 11 e, 11 b, 11 g$. When the XRD data of tris(thio)substituted nitrodienes were investigated, it was shown that the third thiolate anion was replaced with the chlorine atom on C-3 carbon atom. ${ }^{29}$

The 1, 1, 3, 4-tetrachloro-4-bromo-2-nitro-1, 3butadiene 2 reacts at the terminal C-1 carbon atom to yield the product of compound $\mathbf{1 5}$. Isolation and identification proved that a cyclization reaction had taken place, yielding compound 15.

\subsection{Discussion on NMR, IR and MS}

We realized that there were some broad peaks corresponding to the methylene protons of piperazine derivatives when we characterized the new $\mathrm{N}, \mathrm{S}$ substituted nitrodienes via the NMR technique. The piperazine protons are changing from axial to equatorial. At room temperature, there were broad peaks corresponding to piperazine protons in the ${ }^{1} \mathrm{H}-\mathrm{NMR}$. The ${ }^{1} \mathrm{H}-\mathrm{NMR}$ spectra of $\mathbf{7 a}-\mathbf{f}$ showed the piperazine ring, piperazine protons are observed as broad singlets between $\delta 2.5-3.2 \mathrm{ppm}$ and $3.5-3.8 \mathrm{ppm}$. The proton NMR data of 7a-f showed typical ppm values and splitting patterns ( $\mathrm{AA}^{\prime} \mathrm{BB}^{\prime}$-system of the aromatic protons) within the range of $6.6-7.5 \mathrm{ppm}$ and $0.8-3.0 \mathrm{ppm}$ for the methyl protons, respectively. In addition, alkyl units of $7 \mathbf{a}, 7 \mathbf{b}, 7 \mathbf{e}$ and $7 \mathbf{f}$ have been assigned by appropriate incremental shifts owing to substituted benzene derivatives. $^{30}$

The ${ }^{1} \mathrm{H}-\mathrm{NMR}$ spectra of $\mathbf{4 d}$ showed thiomorpholine ring as triplet at $\delta 2.65$ and broad singlet $3.4 \mathrm{ppm}$. The ${ }^{13} \mathrm{C}-\mathrm{NMR}$ shifts of the $\mathrm{C}-1$ carbon atoms of N, S-substituted-trichloro-1, 3-butadiene compound 4d appears relatively downfield around $170 \mathrm{ppm}$, whereas the $\mathrm{NO}_{2}$-bearing carbon atoms, $\mathrm{C}-2$, each show their resonance, a broadened less intense peak, at $133 \mathrm{ppm}$. The individual C-3 and C-4 carbons provide chemical shift values around $118 \mathrm{ppm}$ and $124 \mathrm{ppm}$, respectively.

In the MS[+ESI] mass spectrum of the compound 7f, the respective molecular ion peak was observed at $m / z 678[\mathrm{M}]^{+}$(figure 2). Also, other fragments were observed at $m / z 739[\mathrm{M}+\mathrm{Na}+\mathrm{K}]^{+}, 701[\mathrm{M}+\mathrm{Na}]^{+}$. In MS/MS[+ESI], major fragment $\mathrm{F}_{1}$ of compound 7f was found at $\mathrm{m} / \mathrm{z} 528$ (figure 3). It is likely that this corresponds to the one nitronium $\left[\mathrm{NO}_{2}\right]^{+}$ and three chlorine $[\mathrm{Cl}]^{+}$ions. The respective molecular ion peaks were observed at $m / z 642[\mathrm{M}-\mathrm{Cl}]^{+}$, 


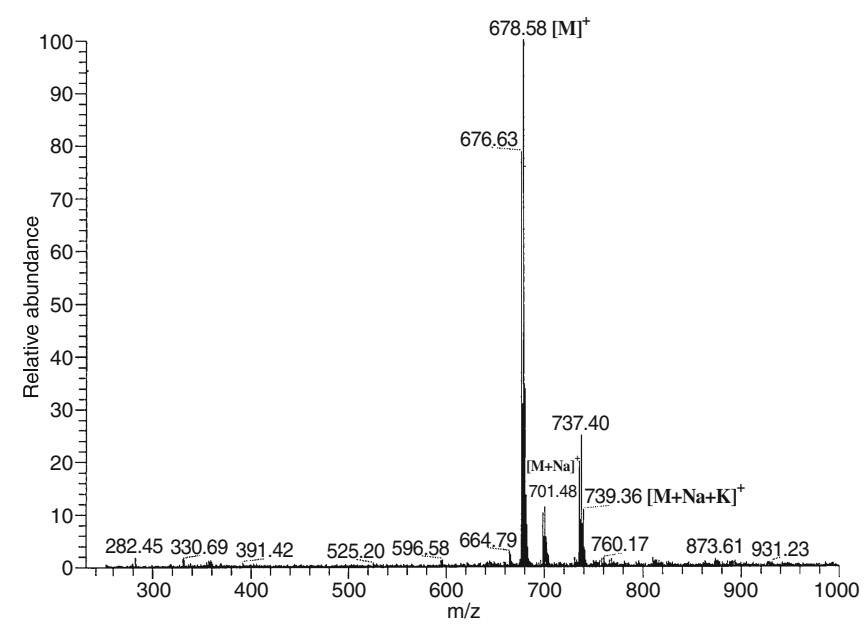

Figure 2. MS[+ESI] spectra of compound $\mathbf{7 f}$.

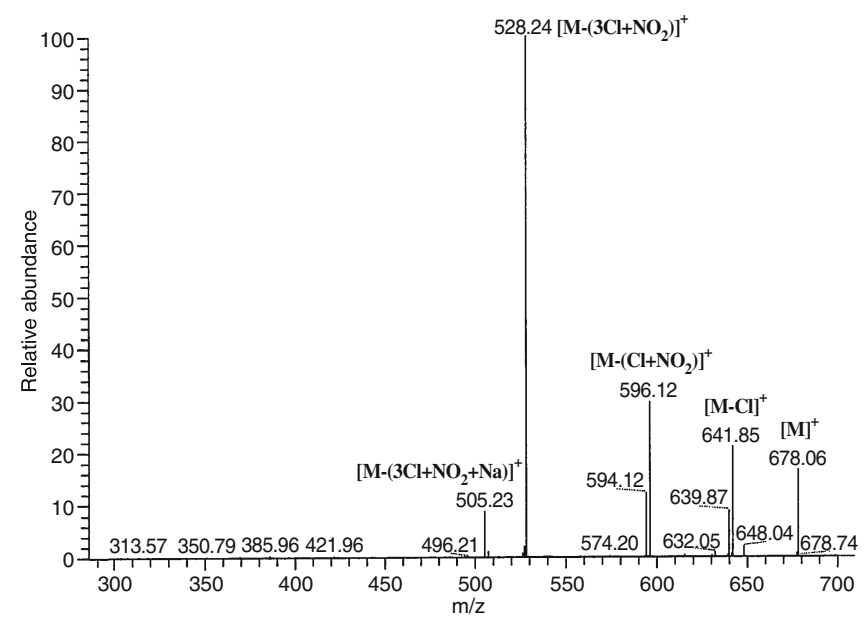

Figure 3. MS/MS[+ESI] spectra of compound $7 \mathbf{f}$.

$596\left[\mathrm{M}-\left(\mathrm{Cl}+\mathrm{NO}_{2}\right)\right]^{+}, 505\left[\mathrm{M}-\left(3 \mathrm{Cl}+\mathrm{NO}_{2}+\mathrm{Na}\right)\right]^{+}(\mathrm{fig}-$ ure 3). The results of MS[+ESI] mass spectrum of all the remaining compounds agree well with corresponding fragments in similar compounds.

Some characteristic bands in the IR spectra of all the compounds should be mentioned: The $\mathrm{C}=\mathrm{C}$ stretching band is observed within the range of 1429 (asymmetric stretching) and $1696 \mathrm{~cm}^{-1}$ (symmetric stretching), and the $\mathrm{NO}_{2}$ groups are observed in the range of 1255 (asymmetric stretching) and $1442 \mathrm{~cm}^{-1}$ (symmetric stretching). In the IR spectra of $\mathbf{8 h}$ and $\mathbf{1 4 h}$, no band is observed in the region $3200-3450 \mathrm{~cm}^{-1}$, attributable to the stretching vibration of the bonded $\mathrm{NH}$ group, indicating the formation of a disubstituted butadienyl homopiperazine compounds. The $\mathrm{N}-\mathrm{H}$ band were observed at 3849 and $3784 \mathrm{~cm}^{-1}$ for compounds $\mathbf{9 h}$ and $\mathbf{1 3 h}$, respectively.

The FT-IR spectra of 10e and 11e showed characteristic $(-\mathrm{OH})$ bands at 3338 and $3362 \mathrm{~cm}^{-1}$ and there were singlet peaks corresponding to $(-\mathrm{OH})$ protons at 3.0 and $3.1 \mathrm{ppm}$ in the ${ }^{1} \mathrm{H}-\mathrm{NMR}$ spectrum. The ${ }^{13} \mathrm{C}$ NMR shifts of the butadiene unit of di(thio)substitutedbutadiene compound 10e were at 119.28, 122.13, 126.64 and $161.99 \mathrm{ppm}$; with $117.28123 .13,155.64$ and $162.99 \mathrm{ppm}$ for tris(thio)substituted-butadiene compound 11e, respectively.

\subsection{Crystal structure of disubstituted butadienyl homopiperazine compound (6b)}

The crystal structure of compound $\mathbf{8 c}$ is shown in figure 3. The titled compound, $\mathrm{C}_{21} \mathrm{H}_{28} \mathrm{Cl}_{6} \mathrm{~N}_{4} \mathrm{O}_{4} \mathrm{~S}_{2}$, contains the expected N, S-substituted butadienyl skeleton, an alkylsulphanyl chain and homopiperazine ring. The butadiene group I has a conformation closer to cisoid than to transoid, the $\mathrm{C} 1-\mathrm{C} 2-\mathrm{C} 3-\mathrm{C} 4$ torsion angle being $-56.0(1)^{\circ}$. The torsion angle of the butadiene group II is $64.0(1)^{\circ}$. Butadiene units are not planar as would be if the two double bonds were fully conjugated. Noncoplanar structure of the butadiene fragments of this molecule and the clear single bonds of the C2-C3 and C15-C16 bonds (1.44(1) $\AA$ and 1.42(2) $\AA$, respectively) indicate the lack of delocalization of $\pi$-electron density in the butadiene chains, which is apparently one of the major reasons for the inertness of polychlorobutadienes and their functional derivatives with nonplanar molecular structure relative to 1,4 -addition. The $\mathrm{C}-\mathrm{C}$ bond lengths within the butadiene unit are similar to those in related compounds. ${ }^{31}$

Homopiperazine is in a boat conformation and not planar with maximum deviation of $0.2055 \AA$. The boat conformation of homopiperazine ring agrees well with corresponding conformation in a similar compound. ${ }^{32}$ Crystal structure of $\mathbf{8 c}$ is the first example of disubstituted butadienyl homopiperazine compound. It is shown that the N, S-substituted polyhalogene-1, 3butadiene compound (butadiene group I and II) were $E$-isomers, in figure 1.

\section{Conclusion}

With the realization of these reactions applying different nucleophiles (thiols, amines, and multifunctional nucleophiles), a highly useful and efficient synthetic methodology is developed. The significance of this project consists in the shortest possible access to highly functionalized sulphur and heterocyclic compounds from very simple precursor in a one-pot operation. Reactions of 'polyhalogenated nitrobuta-1, 3dienes' continue to evolve, with many new possibilities reported during the last decade. In particular, we may 
propose that these reactions would enable to synthesize any fundamentally desired compounds (a) in high yields and (b) efficiently (in as few steps as possible). The goal of this study was to synthesize and characterize new S-, S, S-, S, S, S-, and N,S-substituted-2nitro-polyhalo-1, 3-butadiene compounds $\mathbf{4 d}, \mathbf{4 h}, \mathbf{5 d}$, 7a-f, 8e, 8-9h, 10e, 11b, 11g, 12h and 13h. Their structures were determined by using micro-analysis, FTIR, ${ }^{1} \mathrm{H}-\mathrm{NMR},{ }^{13} \mathrm{C}-\mathrm{NMR}$ and MS. The crystal structure of compound $\mathbf{8 c}$ was determined by X-ray diffraction method.

\section{Acknowledgement}

Authors thank the Research Project Fund of Istanbul University for financial support.

\section{References}

1. Ibis C, Goksel F S and Aydinli G 2003 Phosphorus Sulfur Silicon Relat. Elem. 178777

2. Zapol'skii V A, Nutz E, Namyslo J C, Adam A E W and Kaufmann D E 2006 Synthesis 172927

3. Knapp J S and Brown V R 1988 Int. Biodeterior. 25 299

4. Knapp J S, Emtiazi G, Yusoff S and Heron S T 1996 Lett. Appl. Microbiol. 23334

5. Dmitrenko G N, Gvozdyak P I and Udod V M 1987 Khim. Teknol. Vody 9442

6. Cech J S, Hartman P, Slosare M and Chudoba J 1988 Appl. Environ. Microbiol. 54619

7. Barbachyn M R, Hutchinson D K, Brickner S J, Cynamon M H, Kilburn J O, Klemens S P, Glickman S E, Grega K C, Hendges S K, Toops D S, Ford C W and Zurenko G E 1996 J. Med. Chem. 39680

8. Romero M, Harrak Y, Basset J, Ginet L, Constant P and Pujol M D 2006 Tetrahedron 629010

9. Lopez-Rodriguez M L, Benhamu B, Morcillo M J, Tejada I, Avila D, Marco I, Schiapparelli L, Frechilla D and Del R 2003 J. Bioorg. Med. Chem. Lett. 133177

10. Lopez-Rodriguez M L, Ayala D, Benhamu B, Morcillo M J and Viso A 2002 Curr. Med. Chem. 9443

11. Gonzalez-Gomez J C, Santana L, Uriarte E, Brea J, Villason M, Loza M I, De Luca M, Rivas M E,
Montenegro G Y and Fontenia J A 2003 Bioorg. Med. Chem. Lett. 13175

12. Cappelli A, Gallelli A, Manini M, Anzini M, Memnuni L, Makovec F, Menziani M C, Alcaro S, Ortuso F and Vomero S 2005 J. Med. Chem. 483564

13. Ibis C, Sayil M C and Ozkok F 2006 Z. Naturforsch. 61(9) 1174

14. Ibis C and Deniz N G 2007 Indian J. Chem. 46(4) 674

15. Ibis C and Tuyun A F 2011 Curr. Org. Synth. 8(6) 861

16. Ibis C and Yildirim H 2011 Phosphorus Sulfur Silicon Relat. Elem. 1862236

17. Ibis C, Deniz N G and Tuyun A F 2010 J. Chem. Crystalogr. 40(4) 353

18. Ibis C and Deniz N G 2008 Indian J. Chem. 47(9) 1407

19. Altomare A, Cascarano G, Giacovazzo C, Guagliardi A, Burla M, Polidori G and Camalli M 1994 J. Appl. Crystalogr. 27435

20. Watkin D J, Prout C K, Carruthers J R and Betteridge J R 1996 Crystals Issue 10 P W Chemical Crystallography Laboratory, Oxford, UK

21. Farrugia L J 1997 J. Appl. Crystalogr. 30565

22. Crystallographic data (excluding structure factors) for the structures in this paper have been deposited in the Cambridge Crystallographic Data Centre as supplementary publication number CCDC 907293 for 8c. Copies of the data can be obtained, free of charge, via www.ccdc.cam.ac.uk/conts/retrieving.html or from the Cambridge Crystallographic Data Centre, CCDC, 12 Union Road, Cambridge CB2 1EZ, UK; fax: +44 1223 336033.E-mail: deposit@ccdc.cam.ac.uk

23. Kaberdin R V, Potkin V I and Zapol'skii V A 1997 Russ. Chem. Rev. 66(10) 827

24. Kaberdin R V, Potkin V I and Zapol'skii V A 1999 Russ. Chem. Rev. 68(9) 765

25. Ibis C, Sayil C and Deniz N G 2006 Acta Crystalogr. E62 0800

26. Ibis C and Yıldırım H 2009 Phosphorus Sulfur Silicon Relat. Elem. 184369

27. Aydınlı G, Sayil C and Ibis C 2010 Spectrosc. Lett. 43 44

28. Ol'dekop Yu A, Kaberdin R V and Potkin V I 1980 Zh. Org. Khim. 16543

29. Ibis C and Deniz N G 2007 Acta Crystalogr. E63 o3058

30. Hesse M, Meier H and Zeeh B 2005 Spektroskopische Methoden in der organischen Chemie 7. Aufl., Thieme

31. Ibis C and Deniz N G 2007 Acta Crystalogr. E63 o1091

32. Ali S, Mukhopadhyay U, Shirvani S M, Thurston J, Whitmire K H and Khokhar A R 2002 Polyhedron 21(1) 125 